\title{
Treatment challenges in the management of relapsed or refractory non-Hodgkin's lymphoma - novel and emerging therapies
}

This article was published in the following Dove Press journal:

Cancer Management and Research

12 September 2013

Number of times this article has been viewed

\section{Mark P Chao}

Institute for Stem Cell Biology and Regenerative Medicine, Stanford University, Stanford, CA, USA
Correspondence: Mark P Chao Institute for Stem Cell Biology and Regenerative Medicine, School of Medicine, Stanford University, 265 Campus Drive - 3rd floor, Stanford, CA 94305, USA

Email mpchao@stanford.edu

\begin{abstract}
Over the last few decades, advances in immunochemotherapy have led to dramatic improvement in the prognosis of non-Hodgkin's lymphoma (NHL). Despite these advances, relapsed and refractory disease represents a major treatment challenge. For both aggressive and indolent subtypes of NHL, there is no standard of care for salvage regimens, with prognosis after relapse remaining relatively poor. Nevertheless, there are multiple emerging classes of targeted therapies for relapsed/refractory disease, including monoclonal antibodies, antibodydrug conjugates, radioimmunotherapy, small-molecule inhibitors of cell-growth pathways, and novel chemotherapy agents. This review will discuss treatment challenges of NHL, current available salvage regimens for relapsed/refractory NHL, and the safety and efficacy of novel emerging therapies.
\end{abstract}

Keywords: non-Hodgkin's lymphoma, relapsed/refractory disease, novel therapies

\section{Introduction to non-Hodgkin's lymphoma: treatment challenges}

Non-Hodgkin's lymphoma (NHL) is the fifth most common cancer in the US, where its incidence has been increasing over the last three decades. ${ }^{1}$ NHL comprises a group of clinically and biologically diverse diseases, which range from indolent to aggressive clinical courses. Indolent subtypes include but are not limited to follicular lymphoma (FL), marginal zone lymphoma (MZL), cutaneous T-cell lymphoma (CTCL), small-cell lymphocytic lymphoma/chronic lymphocytic leukemia (SLL/CLL), and lymphoblastic lymphoma, which make up $30 \%$ of all NHL cases in the US. Aggressive subtypes include diffuse large B-cell lymphoma (DLBCL), peripheral T-cell lymphoma (PTCL), and mantle-cell lymphoma (MCL). Among aggressive subtypes of NHL, DLBCL is the most prevalent, and represents approximately $30 \%$ of all NHL diagnoses in adults. ${ }^{2}$ Despite treatment advances in the last three decades with the use of combination immunotherapy, a significant fraction of patients relapse or are refractory to these treatments. While many treatment difficulties exist in NHL, rituximab resistance and refractory/relapsed disease represent current and emerging challenges.

\section{Rituximab resistance}

Within the last two decades, the development and use of the monoclonal antibody (mAb) rituximab has dramatically improved the prognosis of NHL patients, and has been the standard of care in front-line treatment regimens. Standard front-line chemotherapy includes rituximab with cyclophosphamide, doxorubicin, vincristine, and prednisone 
(R-CHOP), with expected 5-year and 10-year overall survival (OS) rates of $58 \%$ and $43.5 \%$, respectively. ${ }^{3}$ Rituximab is a chimeric $\mathrm{mAb}$ targeting CD20, a cell-surface marker present on B-lineage cells and consequently expressed on many B-cell lymphoma subtypes. Multiple lines of evidence indicate that rituximab acts in part by engaging Fc receptors on immune effector cells, such as natural killer cells and macrophages, and stimulates such effector functions as antibody-dependent cellular cytotoxicity (ADCC), complement-dependent cytotoxicity (CDC), and induction of apoptosis. ${ }^{4}$ While therapeutic outcomes has dramatically improved in the post-rituximab era, there is increasing evidence of rituximab resistance. Clinical resistance to rituximab is generally defined as a lack of response to a rituximab-containing treatment regimen or clinical progression after 6 months of such a regimen. Diminished response rates to rituximab in patients with prior rituximab treatments have been observed in multiple NHL subtypes. In patients with relapsed FL or low-grade NHL who had previously received single agent rituximab, only $40 \%$ of patients responded with rituximab retreatment. ${ }^{5}$ In one study of relapsed/refractory DLBCL, patients with and without prior rituximab exposure were treated with salvage chemotherapy followed by stem-cell transplantation. Complete response (CR) rates after salvage chemotherapy were lower in patients receiving prior rituximab compared to naïve patients ( $29 \%$ vs $44 \%$ ), although this difference was not significant in multivariate analysis. ${ }^{6}$ However, patients with prior rituximab treatment had significantly worse progression-free survival (PFS) than patients who were rituximab-naïve (17\% vs 57\%). Prior rituximab treatment was an independent adverse prognostic factor for survival. In the Collaborative Trial in Relapsed Aggressive Lymphoma study, relapsed/refractory DLBCL patients who were previously treated with rituximab also had a worse outcome when retreated with rituximab-containing therapies. ${ }^{7}$ Poorer outcomes were specifically seen in previously treated rituximab patients who relapsed or progressed during the first year. These data and others highlight the clinical concern that salvage regimens for relapsed/refractory patients may not be as effective in the era of rituximab usage in front-line regimens. Thus, overcoming rituximab resistance has been a major focus of recent therapeutic development. Several mechanisms of rituximab resistance have been postulated. These include resistance in antibody effector mechanisms (ADCC, CDC, and induction of apoptosis), Fc-receptor polymorphisms, downregulation or loss of CD20 expression, and altered antibody pharmacokinetics. ${ }^{8}$ To address these issues, one major treatment strategy has been the development of novel anti-CD20 antibodies that more effectively engage immune effectors and bind CD20 with higher avidity. Several of these next-generation anti-CD20 antibodies are in late-stage clinical development. Other nonantibody therapies have also been demonstrated to enhance CD20 expression changes and/or sensitize tumor cells to rituximab in the hope of overcoming rituximab resistance. Through potential epigenetic regulation of CD20 expression, histone deacetylase inhibitors (HDACis) have been shown to increase CD20 expression and enhance rituximab cytotoxicity in vitro. ${ }^{9}$ Other therapeutic agents, including temsirolimus, bortezomib, and Bcl-2 inhibitors, have also been shown to sensitize lymphoma cells to rituximab in preclinical studies. A more extensive review of these agents and anti-CD20 antibodies will be undertaken later.

\section{Relapsed and refractory NHL}

Response rates to conventional chemotherapy are generally greater than 50\%; however, most patients eventually relapse. A brief survey of treatment options for refractory DLBCL, PTCL, CLL, FL, and MCL will be discussed.

While no standard of care exists for salvage regimens, a number of regimens have been studied as options for salvage therapy (Figure 1). For DLBCL, a common salvage regimen includes ifosfamide, carboplatin, and etoposide (ICE), which has produced an overall response rate (ORR) of $66 \% .{ }^{10}$ With the addition of rituximab, CR rates improved from $24 \%$ to $53 \%$ without increased toxicity. ${ }^{11}$ Rituximab, etoposide, methylprednisone, cytarabine, and cisplatin (R-ESHAP) achieved an ORR of $73 \%$, representing another common salvage regimen. ${ }^{6}$ In PTCL, treatment options include single- and multiagent chemotherapy, with the exception of rituximab, given the lack of CD20 expression on T-cell lymphomas. Relapse rates are high due to low efficacy of first-line regimens and a relative dearth of large-scale trials for refractory PTCL. Thus, the discovery of novel treatments for PTCL is a high priority. In CLL, the majority of refractory patients are unresponsive to fludarabine, a cornerstone of first-line therapy. In the era of single-agent fludarabine, up to $37 \%$ of patients were found to be fludarabine-refractory, which has decreased to less than $10 \%$ with the addition of rituximab-containing front-line therapies. ${ }^{12,13}$ Genetic abnormalities, including deletion in the short arm of chromosome 17 (del[17p]) and in the long arm of chromosome 11 (del[11q]), are associated with poor response to fludarabine and other agents. ${ }^{14,15}$ Salvage regimens are stratified based on genetic subtype, and include mAbs (alemtuzumab and ofatumumab) and rituximab-containing combination chemotherapies. Salvage options for FL include rituximab alone or in combination with alkylating agents, radioimmunotherapy, and other agents not used in first-line therapy. For patients who respond to salvage immunochemotherapy, maintenance therapy with rituximab has been shown to reduce 


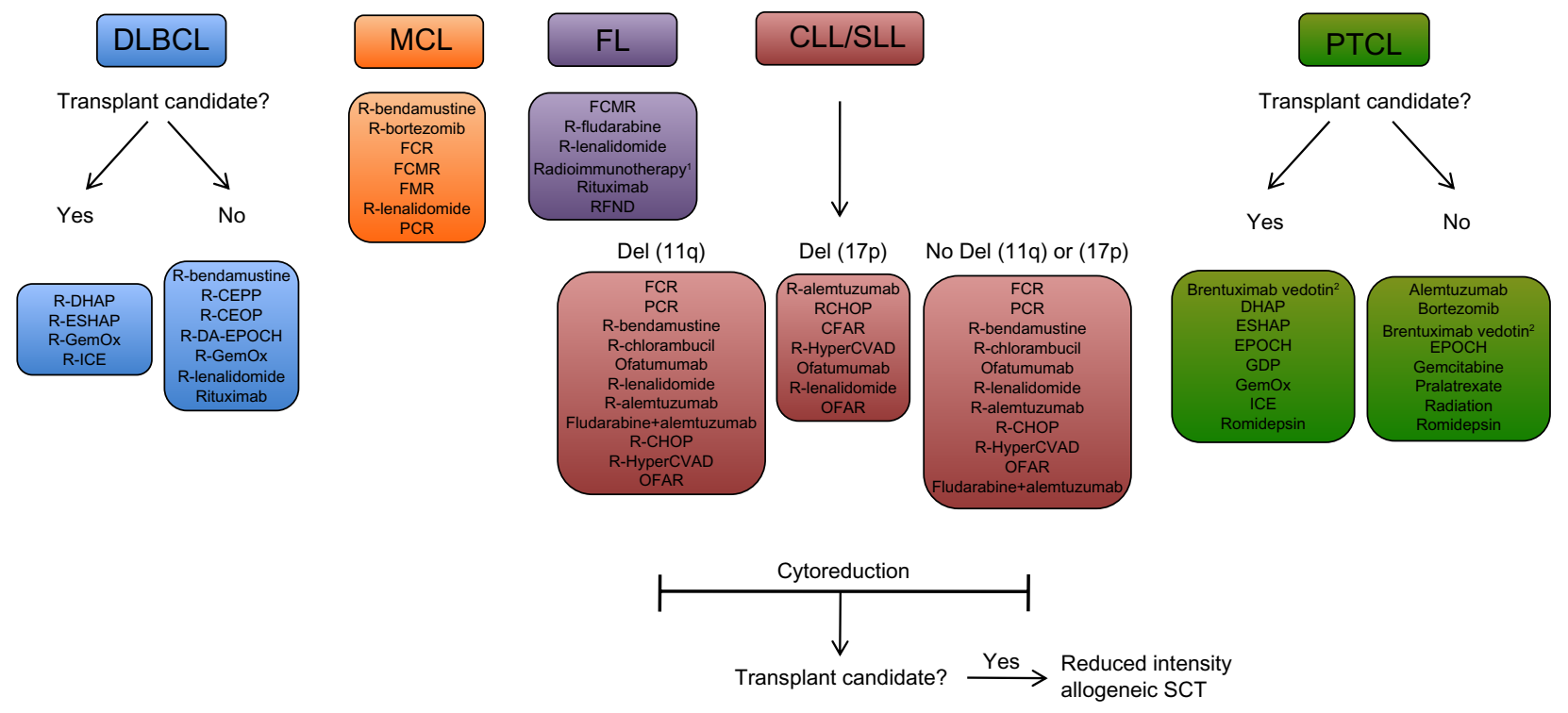

Figure I Examples of salvage therapy regimens for refractory $\mathrm{NHL}$.

Notes: 'Radiommunotherapy options include ${ }^{131} \mathrm{I}$-tositumomab and ${ }^{90} \mathrm{Y}$-ibritumomab tiuxetan; ${ }^{2}$ therapy for systemic ALCL, excluding primary cutaneous ALCL. Treatment options, stratification, and abbreviations are based on NCCN Clinical Practice Guidelines In Oncology (NCCN Guidelines ${ }^{\circledR}$ ) for Non-Hodgkin's Lymphomas V.I.20I3. Not all treatment options included in the NCCN Guidelines are shown above. Reproduced/Adapted with permission from the NCCN Clinical Practice Guidelines in Oncology (NCCN Guidelines ${ }^{\circledR}$ ) for Non-Hodgkin's Lymphomas V.I.20I3. ( 2013 National Comprehensive Cancer Network, Inc. All rights reserved. The NCCN Guidelines ${ }^{\circledR}$ and illustrations herein may not be reproduced in any form for any purpose without the express written permission of the NCCN. To view the most recent and complete version of the NCCN Guidelines, go online to NCCN.org. National Comprehensive Cancer Network ${ }^{\circledR}, \mathrm{NCCN}^{\circledR}$, NCCN Guidelines ${ }^{\circledR}$, and all other NCCN Content are trademarks owned by the National Comprehensive Cancer Network, Inc. ${ }^{131}$

Abbreviations: CEOP, cyclophosphamide, etoposide, vincristine, prednisone; CEPP, cyclophosphamide, etoposide, prednisone, procarbazine; CFAR, cyclophosphamide, fludarabine, alemtuzumab, rituximab; CHOP, cyclophosphamide, doxorubicin, vincristine, prednisone; CLL/SLL, chronic lymphocytic leukemia/small lymphocytic lymphoma; DA-EPOCH, doseadjusted etoposide, prednisone, vincristine, cyclophosphamide, doxorubicin; DHAP, dexamethasone, cisplastine, cytarabine; DLBCL, diffuse large b-cell lymphoma; ESHAP, etoposide, methylprednisolone, cytarabine, cisplatin; FCR, fludarabine, cyclophosphamide, rituximab; FCMR, fludarabine, cyclophosphamide, mitoxantrone, rituximab; FL, follicular lymphoma; FMR, fludarabine, mitoxantrone, rituximab; GDP, gemcitabine, dexamethasone, cisplatin; GemOx, gemcitabine, oxaliplatin; HyperCVAD, fractionated cyclophosphamide, vincristine, doxorubicin, dexamethasone; ICE, ifosphamide, carboplatin, etoposide; MCL, mantle cell lymphoma; OFAR, oxaliplatin, fludarabine, cytarabine, rituximab; PCR, pentostatin, cyclophosphamide, rituximab; PTCL, peripheral T-cell lymphoma; R, rituximab; RFND, rituximab, fludarabine, mitoxantrone, dexamethasone; SCT, stem cell transplant.

the risk of progression and mortality. ${ }^{16}$ For MCL, rituximab in combination with chemotherapies, including bendamustine and fludarabine, have been utilized. Newer agents, including the proteosome inhibitor bortezomib and the thalidomide derivative lenalidomide, have been increasingly incorporated into second-line regimens.

Several factors are considered when selecting a salvage regimen. For aggressive lymphoma subtypes, the decision on therapy is based on whether patients are candidate for stemcell transplants. Those patients that are not candidates (due to age, poor performance status, or significant comorbidities) undergo chemotherapy with a goal of minimizing toxicity. However, prognosis is poor among these patients, with expected survival of less than 1 year. ${ }^{17}$ In DLBCL, autologous hematopoietic stem-cell transplant (auto-SCT) has become the standard of care for patients in first relapse. Based on the landmark Parma trial, relapsed NHL patients were randomized to either conventional chemotherapy or high-dose chemotherapy followed by auto-SCT, with a significantly more favorable response and event-free survival rate in the auto-SCT group. ${ }^{18}$ The use of allogeneic hematopoietic SCT (allo-SCT) compared to auto-SCT has been generally limited.
While allo-SCT has led to lower relapse rates compared to auto-SCT, the myeloablative pretransplant regimen has led to increased treatment-related fatalities. ${ }^{19}$ Treatment-related mortality can reach up to $25 \%$ with allogeneic transplantation, thus precluding its use in many patients. ${ }^{20}$ While autoSCT has a clear role in DLBCL, transplantation is not as clear for other NHL subtypes. Prior prospective studies have shown mixed benefit for extending survival in relapsed FL. ${ }^{21}$ For CLL, SCT has been used in fludarabine-refractory patients. In contrast to other NHL subtypes, allo-SCT is used for CLL, as auto-SCT has not been shown to have curative potential. ${ }^{22}$ Reduced-intensity conditioning regimens for allo-SCT are currently used, given high treatment-related mortality rates observed in myeloablative conditioning. ${ }^{22}$ Cytoreduction with salvage therapy followed by consolidation allo-SCT is often the goal (Figure 1). The role of transplantation is not as well described in other less common NHL subtypes, including T-cell lymphomas and MCL, mainly due to difficulty in patient accrual for large prospective trials. Given the promising efficacy of emerging novel antibody and other targeted therapies for these NHL subtypes, it is possible that stem-cell transplantation will play a more limited role in the future. 


\section{Novel and emerging therapies for NHL}

Over the last decade, a multitude of therapies have entered clinical development for NHL. These therapies range from novel mAbs (including next-generation anti-CD20 mAbs), antibody-drug conjugates (ADCs), radioimmunotherapy, small-molecule inhibitors targeting cell survival and growth pathways, and novel chemotherapies (Figure 2). Many of these therapies have shown considerable promise, providing significant response rates in heavily pretreated NHL patients. Therapeutic agents in early to late-phase clinical trials will be reviewed here.

\section{Monoclonal antibody therapies Anti-CD20 antibodies}

With the emergence of rituximab resistance, next-generation anti-CD20 mAbs have been developed to augment effectorfunction potency and increase CD20 binding avidity. Secondand third-generation anti-CD20 antibodies have been developed and are in clinical trials. Second-generation antibodies all possess fully human immunoglobulin $\mathrm{G}$ to reduce immunogenicity, and include ocrelizumab, veltuzumab, and ofatumumab. Thirdgeneration antibodies are humanized, and have an engineered
Fc region to improve therapeutic effector functions. These include AME-133v, GA101, TRU-015, PRO13192, and others. Clinical data on late-stage second-generation anti-CD20 antibodies will be reviewed here (Table 1). Anti-CD20 antibodies in early phase clinical development, including third-generation antibodies, are reviewed elsewhere. ${ }^{23,24}$

\section{Ofatumumab}

Ofatumumab is the first humanized anti-CD20 mAb approved in the US and Europe. It is a second-generation anti-CD20 mAb that binds both small and large loops of the CD20 surface epitope, whereas rituximab binds to the large loop alone. ${ }^{25}$ Unique features of ofatumumab include high CD20 epitope sensitivity, slow off rate, and high CDC activity. ${ }^{26}$ In vitro, ofatumumab induces stronger CDC in cell lines that are rituximab-resistant. ${ }^{27}$ Ofatumumab was first evaluated as a monotherapy in a phase I/II trial on heavily pretreated patients with relapsed/refractory CLL. Ofatumumab was very well tolerated, with an ORR of $50 \%$ at the highest dose administered. ${ }^{28}$ A subsequent landmark phase II trial of ofatumumab in fludarabine and alemtuzumabrefractory patients demonstrated an ORR of $47 \%-58 \%$ in both groups, with the most common adverse events being

\section{Cell signaling pathway small molecule inhibitors}

$\begin{array}{cll}\text { PI3K/AKT/mTOR inhibitors } & \text { BTK inhibitors } & \text { Syk inhibitors } \\ \text { GS-1101, perifosine, temsirolimus, everolimus } & \text { Ibrutinib } & \text { Fostamatinib disodium }\end{array}$

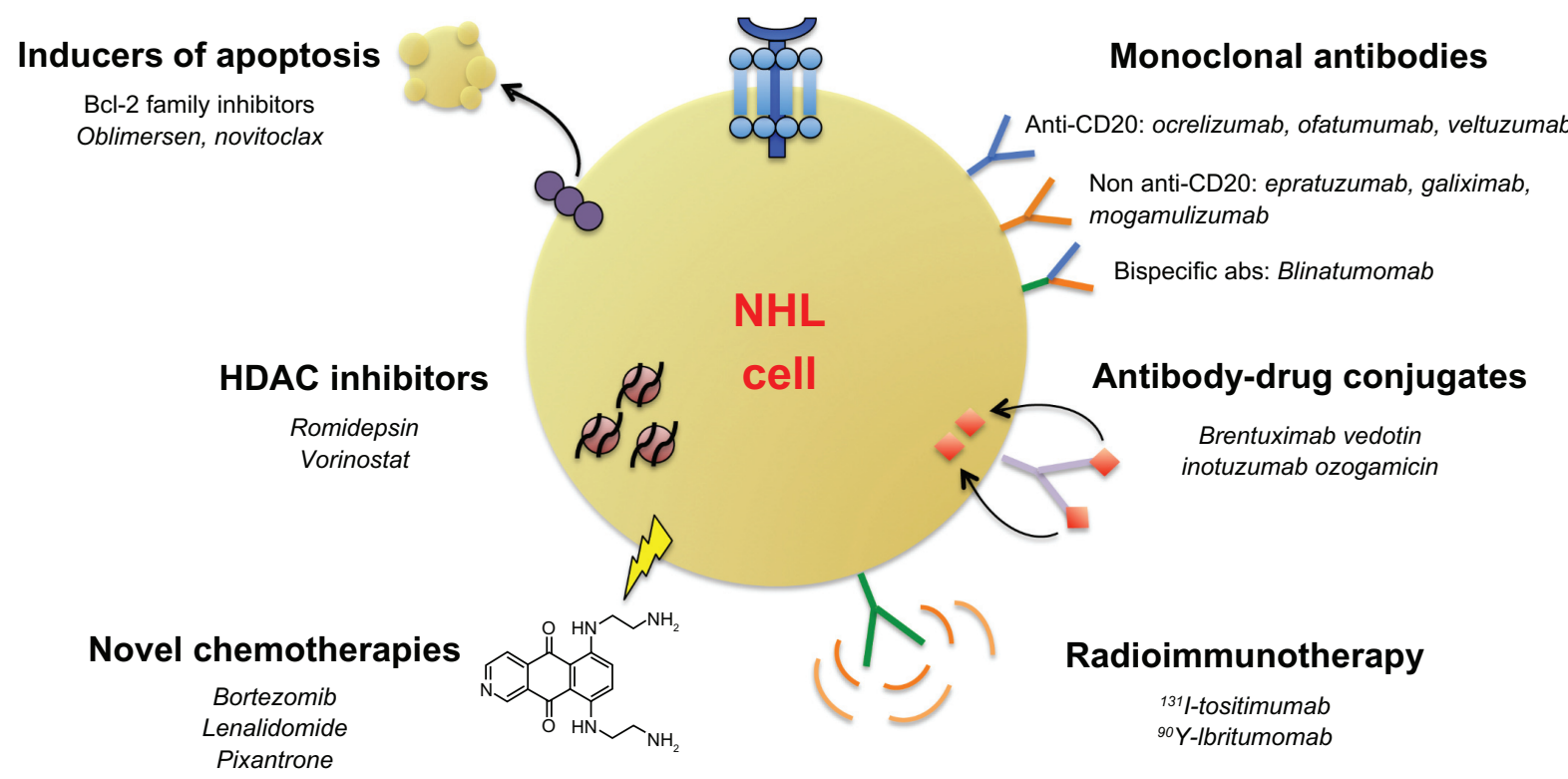

Figure 2 Emerging therapeutic options in relapsed/refractory NHL. Numerous novel classes of drugs are emerging as therapeutic options in relapsed or refractory NHL. These classes include monoclonal antibodies (both next-generation anti-CD20 and non-anti-CD20 antibodies), antibody-drug conjugates, antibodies conjugated to radioactive isotopes, small-molecule inhibitors of key cell-signaling and apoptotic pathways, HDAC inhibitors, and toxicity-reducing chemotherapies.

Abbreviations: abs, antibodies; Bcl-2, B-cell lymphoma 2; BTK, Bruton tyrosine kinase; HDAC, histone deacetylase; mTOR, mammalian target of rapamycin; NHL, nonHodgkin's lymphoma; Syk, spleen tyrosine kinase; PI3K, phosphoinositide 3-kinase. 


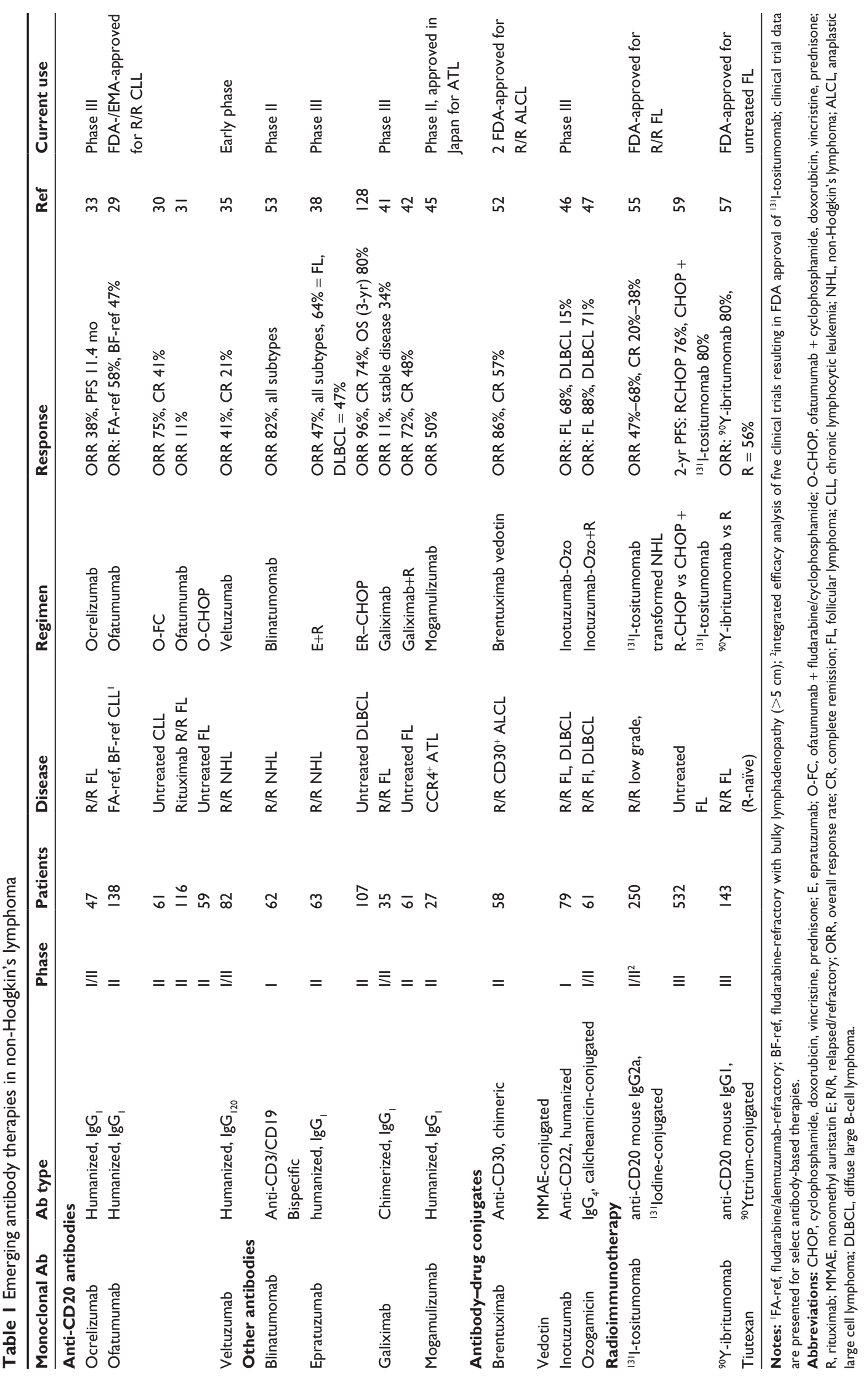


grade 1-2 infections or infusion reactions. ${ }^{29}$ Based on these results, the FDA approved ofatumumab for the treatment of CLL patients refractory to fludarabine and alemtuzumab. Ofatumumab has also been evaluated in combination with front-line chemotherapy for patients with untreated CLL. ${ }^{30}$ Patients received ofatumumab in combination with fludarabine and cyclophosphamide, yielding an ORR and CR across all patients of $75 \%$ and $41 \%$, respectively. Ofatumumab has also been studied as a monotherapy in FL. While ofatumumab was well tolerated in the initial phase I/II trial, a subsequent phase II trial was conducted in rituximab-refractory patients and demonstrated a relatively poor response rate of $11 \% .{ }^{31}$ This observation suggested that ofatumumab might be more effective in a combination strategy in refractory FL. Indeed, in untreated FL patients, ofatumumab in combination with CHOP demonstrated an ORR of greater than $90 \%$ with a CR rate over $75 \%{ }^{32}$ Combination therapy with ofatumumab is also being studied in late-phase trials for FL and DLBCL, with hopes of gaining FDA approval for these subtypes.

\section{Ocrelizumab}

Ocrelizumab is a second-generation anti-CD20 antibody that differs from rituximab at several positions within the complementary-determining regions of the light- and heavychain variable regions, leading to increased affinity for the FcyRIIIa receptor and enhanced ADCC.${ }^{33}$ Ocrelizumab has been evaluated in a phase I/II study in relapsed/refractory FL patients that received prior rituximab therapy. ${ }^{33}$ Treatment was well tolerated, with grade $3 / 4$ toxicities occurring in only $9 \%$ of patients and an ORR of $38 \%$. While initial studies are encouraging, its clinical development for autoimmune diseases (an alternative indication) was suspended after excess deaths due to opportunistic infections, which may cause concern in future lymphoma studies.

\section{Veltuzumab}

Veltuzumab is a second-generation anti-CD20 antibody constructed on the framework regions of the anti-CD22 antibody epratuzumab, with complementary determining regions identical to rituximab, with the exception of a single amino acid chain substitution. In vitro, ADCC efficacy is similar to rituximab; however, veltuzumab has been reported to posses slower off rates and increased CDC. ${ }^{34}$ Veltuzumab was evaluated in a phase I/II study of relapsed/refractory NHL patients. ${ }^{35}$ ORR across all subtypes was $41 \%$, ranging from $43 \%$ to $83 \%$ in DLBCL and MZL patients, respectively. Therapy was well tolerated, with no grade $3 / 4$ adverse events. Additional early phase trials are ongoing.

\section{Other monoclonal antibodies}

\section{Epratuzumab}

Epratuzumab is a humanized anti-CD22 antibody that has been clinically studied in indolent and aggressive NHL. CD22 is a cell-surface antigen expressed on mature $\mathrm{B}$ lymphocytes and regulates B-cell function and survival. CD22 expression has been observed in $60 \%-80 \%$ of B-cell malignancies and over $90 \%$ in FL and DLBCL. ${ }^{36}$ Epratuzumab has also been investigated in combination-therapy strategies, most notably with rituximab. Initial phase I data demonstrated that this combination is well tolerated.$^{37} \mathrm{~A}$ phase II trial of combination therapy with rituximab and epratuzumab in relapsed/ refractory NHL patients $(23 \%$ receiving prior rituximab therapy) demonstrated favorable responses (ORR of $47 \%$ and CR of 22\%), with the best responses seen in FL and DLBCL (Table 1) ${ }^{38}$ Epratuzumab and rituximab have also been evaluated in front-line therapy. When these two antibodies were combined with CHOP chemotherapy in untreated DLBCL patients, ORR was $96 \%$, with a CR of $72 \%$ and 1 -year PFS of $82 \%$. Toxicity was similar to standard R-CHOP. These results suggest that epratuzumab with R-CHOP may be a promising front-line therapy employing combination antibodies. Based on these data, phase III trials have been initiated.

\section{Galiximab}

Galiximab is a chimeric anti-CD80 mAb initially evaluated in psoriasis in phase I/II trials, where it was found to have minimal side effects. ${ }^{39} \mathrm{CD} 80$ is a protein found on activated $\mathrm{B}$ cells that provides a costimulatory signal necessary for T-cell activation. It is constitutively expressed in most B-cell lymphomas. ${ }^{40}$ The initial phase I/II study of galiximab as a single agent was initiated in relapsed/refractory FL, with an ORR of $11 \%$ (Table 1 ). ${ }^{41}$ Sixty percent of patients experienced adverse events related to galiximab, but most were low-grade toxicities of fatigue, nausea, and headache. Hematologic toxicities were rare. Galiximab in combination with rituximab has also been evaluated as first-line treatment for FL in the Cancer and Leukemia Group B's study 50402. ${ }^{42}$ Untreated FL patients received eight cycles of galiximab and rituximab that was well tolerated, with an ORR of $71 \%$ with $48 \%$ of patients achieving CR or unconfirmed CR. Phase III studies are ongoing to evaluate galiximab in combination with rituximab for FL.

\section{Mogamulizumab}

Mogamulizumab is a humanized antibody targeting the chemokine receptor CCR4. CCR4 is a transmembrane receptor selectively expressed on T-helper 2 and regulatory $\mathrm{T}$ cells that is expressed in $90 \%$ of patients with adult T-cell 
leukemia/lymphoma (ATL). ${ }^{43}$ In ATL, CCR4 expression was associated with increased skin infiltration and worse outcomes. Given the relatively few therapies available for relapsed/refractory ATL, CCR4 inhibition has emerged as a promising agent. Mogamulizumab recognizes the $\mathrm{N}$-terminal region of human CCR4 and is defucosylated to enhance ADCC. Mogamulizumab was first investigated in CCR4positive relapsed PTCL (including ATL), demonstrating no dose-limiting toxicities and an ORR of $31 \%$ (Table 1). ${ }^{44} \mathrm{~A}$ subsequent phase II trial of mogamulizumab was conducted in CCR4-positive relapsed ATL. ${ }^{45}$ ORR was $50 \%$, with $100 \%$ response rate in the blood, $63 \%$ in the skin, and $25 \%$ for nodal/extranodal lesions. The most common adverse events were infusion reactions and skin eruptions. However, several cases of Stevens-Johnson syndrome have been reported, with further trials needed to evaluate for safety and efficacy. Nevertheless, mogamulizumab received regulatory approval for use in ATL in Japan in 2012. Clinical trials are ongoing for evaluation of approval in the US and Europe.

\section{Antibody-drug conjugates}

ADCs consist of a monoclonal antibody conjugated to a cytotoxic agent, whereby the mechanism of activity occurs by antigen-antibody binding and subsequent internalization of the cytotoxic drug. Drug conjugates not only enhance therapeutic efficacy of the original antibody but also do so with limited side effects and a wider therapeutic window, given tumor antigen-specific targeting.

\section{Inotuzumab ozogamicin}

Inotuzumab ozogamicin (CMC-544) is a human anti-CD22 immunoglobulin $\mathrm{G}_{4}$ antibody conjugated to the antitumor antibiotic calicheamicin, and is the most studied ADC in NHL. Because CD22 undergoes constitutive endocytosis, it is a well-suited target for intracellular toxin delivery. Given the relatively benign toxicity profile and promising tumor efficacy of the "naked" anti-CD22 epratuzumab, an $\mathrm{ADC}$ to CD22 was developed. In the first-in-human study, inotuzumab ozogamicin was administered to previously treated FL and DLBCL patients, and achieved an ORR of $39 \%$ (68\% in FL and $15 \%$ in DLBCL) (Table 1). ${ }^{46}$ The combination of inotuzumab ozogamicin and rituximab was evaluated in phase I/II trials in relapsed/refractory FL or DLBCL, with a preliminary ORR reported of $88 \%$ for FL and $71 \%$ for DLBCL in the maximal-tolerable-dose cohort. ${ }^{47}$ However, response to prior therapy appears to be a major prognostic factor, as rituximab-refractory patients had ORR rates less than $20 \%$. The most common treatment toxicities of inotuzumab ozogamicin observed in early trials were thrombocytopenia, neutropenia, and leukopenias. Based on these early studies, phase III trials are under way.

\section{Brentuximab vedotin}

CD30 is a cell-membrane receptor involved in nuclear factor- $\kappa \mathrm{B}$ signaling and cell survival, and is widely expressed on Hodgkin's and anaplastic large cell lymphoma (ALCL) cells. ${ }^{48}$ Therapeutic antibodies targeting CD30 have been developed, and while demonstrating impressive preclinical activity in xenograft mouse models, early clinical trials in HL and ALCL have shown only modest efficacy. In addition, dose-limiting pulmonary toxicity has been observed when combined with other chemotherapies. ${ }^{49,50}$ In contrast to the naked anti-CD30 antibody, the ADC brentuximab vedotin (SGN-35), which conjugates anti-CD30 antibody to the antimicrotubule agent monomethyl auristatin E (MMAE), has shown significant activity in HL and ALCL. Antitumor efficacy of brentuximab vedotin stems from both cellular internalization of MMAE and release of free MMAE by tumor cells to surrounding cells. Clinical studies were first initiated in HL, leading to FDA approval for both HL and ALCL. Data on HL are reviewed elsewhere. ${ }^{51}$ Accelerated approval for ALCL was based on a multicenter trial treating CD30-positive systemic ALCL patients with brentuximab vedotin who had previously received front-line multiagent chemotherapy. ${ }^{52}$ ORR was $86 \%$, with a CR rate of $57 \%$ and median duration response of 13.2 months (Table 1). Serious adverse events were reported in $31 \%$ of patients, of which peripheral neuropathy and abdominal pain were the most common. Recently, several cases of progressive multifocal leukoencephalopathy have been reported with brentuximab vedotin use, and while rare, these observations have led to an FDA boxed warning. Caution must also be made when combining brentuximab vedotin with other chemotherapies leading to increased pulmonary toxicity, including bleomycin.

\section{Bispecific antibodies}

Bispecific antibodies consist of an antibody composed of fragments of two monoclonal antibodies that bind separate antigens. Bispecific antibodies have distinct advantages over traditional mAbs, including increased tumor-targeting specificity through binding of two tumor-specific antigens and enhanced cytotoxic efficacy by engagement of multiple effector mechanisms. While several bispecific antibodies have been engineered for anticancer therapy for NHL, only one has proceeded through significant clinical trials: blinatumomab. Blinatumomab is a bispecific antibody targeting CD19 (a 
B-cell marker) and CD3 (a T-cell engager) and belongs to the class of bispecific T-cell engager antibodies whereby tumor antibody-antigen targeting is linked to recruitment of T-cell activity. The first of its class utilized in NHL, blinatumomab targets CD19, which is widely expressed across all NHL subtypes and important for proliferation and survival of $\mathrm{B}$ cells. Targeting of CD19-expressing tumor cells is linked to recruitment and activation of cytotoxic $\mathrm{T}$ cells through simultaneous ligation of $\mathrm{CD} 3$ on the T-cell receptor, leading to T-cell-mediated tumor lysis. Blinatumomab has been clinically studied in both NHL and acute lymphoblastic leukemia. The first phase I study of 62 NHL patients treated with the recommended dose of blinatumomab demonstrated an ORR of $82 \%$ across NHL subtypes, with over $60 \%$ of responders maintaining durable response up to 3 years out from therapy (Table 1). ${ }^{53}$ The most significant adverse events reported were reversible central nervous system toxicities (including encephalopathy, tremor, and aphasia), which has led to discontinuation of the study drug in over $20 \%$ of patients. The mechanism of central nervous system-related events is currently not yet understood. Currently, phase II trials have been initiated in NHL patients. Multiple other bispecific antibodies are undergoing clinical investigation, including 4G7XH22 (anti-CD19/CD30), Bi20 (anti-CD20/CD3), and anti-CD20/CD22 antibodies. ${ }^{23}$

\section{Radioimmunotherapy}

As another therapeutic application of antibodies, radioimmunotherapy employs $\mathrm{mAb}$ or $\mathrm{mAb}$-derived vectors that are chemically conjugated to radioactive isotopes for tumor targeting and delivery. Radioisotope-conjugated antibodies have a key theoretical advantage compared to ADCs and other $\mathrm{mAb}$ therapies in that tumor cells not bound by $\mathrm{mAb}$ (due to low or negative target-antigen expression) can still be eliminated by cytotoxic effects of the surrounding radiation field. For NHL, the FDA has approved two radioimmunoconjugates: ${ }^{131}$ I-tositumomab (Bexxar) and ${ }^{90} \mathrm{Y}$-ibritumomab tiutexan (Zevalin), both of which target the CD20 antigen. In 2003, the FDA approved ${ }^{131}$ I-tositumomab for the treatment of relapsed or refractory FL. In $2009,{ }^{90}$ Y-ibritumomab tiutexan was approved as a front-line consolidation treatment after induction chemotherapy in FL.

Both ${ }^{131} \mathrm{I}$-tositumomab ${ }^{90} \mathrm{Y}$-ibritumomab tiutexan have been extensively studied, and demonstrated high response rates and durable remissions within heavily pretreated populations, with predictable and manageable toxicity. ${ }^{54-58} \mathrm{An}$ integrated analysis of five clinical trials on heavily pretreated indolent lymphoma patients treated with ${ }^{131} \mathrm{I}$-tositumomab demonstrated response rates between $47 \%$ and $68 \%$ and CR rates between $20 \%$ and $38 \%$ (Table 1). ${ }^{55}$ Patients who achieved CR had response durations that lasted years, with median duration not achieved at 5 years. Similar durable response rates for patients with relapsed/refractory FL or DLBCL have also been observed with ${ }^{90}$ Y-ibritumomab tiutexan, with median duration of response over 3 years in patients who responded to treatment. ${ }^{58}$ Both of these drug conjugates have also been evaluated as first-line treatment for FL and other indolent NHL as well as consolidation therapy after initial chemotherapy, with high ORR and CR rates. A recent phase III trial randomizing six cycles of R-CHOP vs CHOP followed by ${ }^{131}$ I-tositumomab demonstrated high rates of PFS, but no difference in PFS or OS between the two groups. ${ }^{59}$ Multiple phase I/II trials have also been initiated to evaluate both radioisotope conjugates with other chemotherapeutic regimens.

While both radioisotope-conjugated antibodies have shown considerable efficacy, do these drugs have acceptable toxicities given the use of radioisotopes? In early and late-phase trials, safety profiles have been favorable, with reversible hematologic effects as the primary toxicity observed. In addition, these antibody conjugates are chimeric antibodies, raising theoretical concern for increased antibody immunogenicity. Nevertheless, human antichimeric and human antimouse antibody formation has been minimal, between $0.05 \%$ and $1 \%$, respectively. ${ }^{60}$ As part of the treatment regimen, predosing with an unlabeled "cold" mAb is used to saturate normal tissue-antigen sinks that highly express CD20 (ie, the spleen), which allows for improved tumor distribution and decreased toxicity. ${ }^{61}$ While radiation-induced secondary malignancies may be a concern with these therapies, no increased incidence of myelodysplastic syndrome (MDS) or acute myeloid leukemia (AML) has been seen. In over 1,000 patients treated with ${ }^{131}$ I-tositumomab monitored from 2 to 5 years from initial treatment, incidence of treatment-related MDS/AML occurred in 3.5\% of prior chemotherapy-treated patients, with no increased incidence in any patients treated with ${ }^{131} \mathrm{I}$-tositumomab as their initial therapy compared to nonradioisotope therapies. ${ }^{62}$ For patients treated with ${ }^{90} \mathrm{Y}$-ibritumomab tiutexan, annualized incidence rates of MDS or AML were $0.3 \%$ from time of diagnosis and $0.7 \%$ from time of radioimmunotherapy. The posttreatment rate is comparable to rates reported after long-term alkylating agents. ${ }^{63}$ In summary, radioimmunotherapy is a highly effective first-line therapy for indolent lymphoma as well as in relapsed refractory NHL, with minimal and acceptable toxicity.

\section{Phosphatidylinositol 3-kinase pathway and other small-molecule inhibitors}

While significant progress has been made in therapeutic antibodies, perhaps the most rapid advances have been made 
in nonantibody therapeutics, including the development of small-molecule inhibitors that modulate key pathways important in NHL pathogenesis. The phosphatidylinositol 3-kinase (PI3K) pathway is a ubiquitous pathway involving a large number of lipid kinases that play critical roles in cell survival, proliferation, metabolism, and angiogenesis. The PI3K pathway is inappropriately activated in many cancers, including NHL, whereby activation is critical for tumor progression and resistance to antitumor drugs. ${ }^{64}$ Within the PI3K pathway, activation of PI3K and its downstream kinases AKT, mammalian target of rapamycin (mTOR), and bruton tyrosine kinase (BTK) has been observed with high frequency in multiple NHL subtypes, most notably in
DLBCL and MCL. ${ }^{65}$ As a result, there has been wide interest in developing selective inhibitors to these components. The development and clinical outcomes of PI3K, AKT, BTK, and mTOR inhibitors will be specifically reviewed here (Figure 3).

\section{Phosphatidylinositol 3-kinase inhibitors}

PI3Ks are divided into three subclasses on the basis of structure, regulation, and lipid-substrate specificity, of which mutations in class I PI3Ks often lead to activation in many cancers. ${ }^{66}$ Within these subclasses, several different isoforms exist, comprised of four different catalytic $\mathrm{p} 110$ subunits $(\alpha, \beta$, $\delta$, and $\gamma$ ), with variable expression observed across cell types.

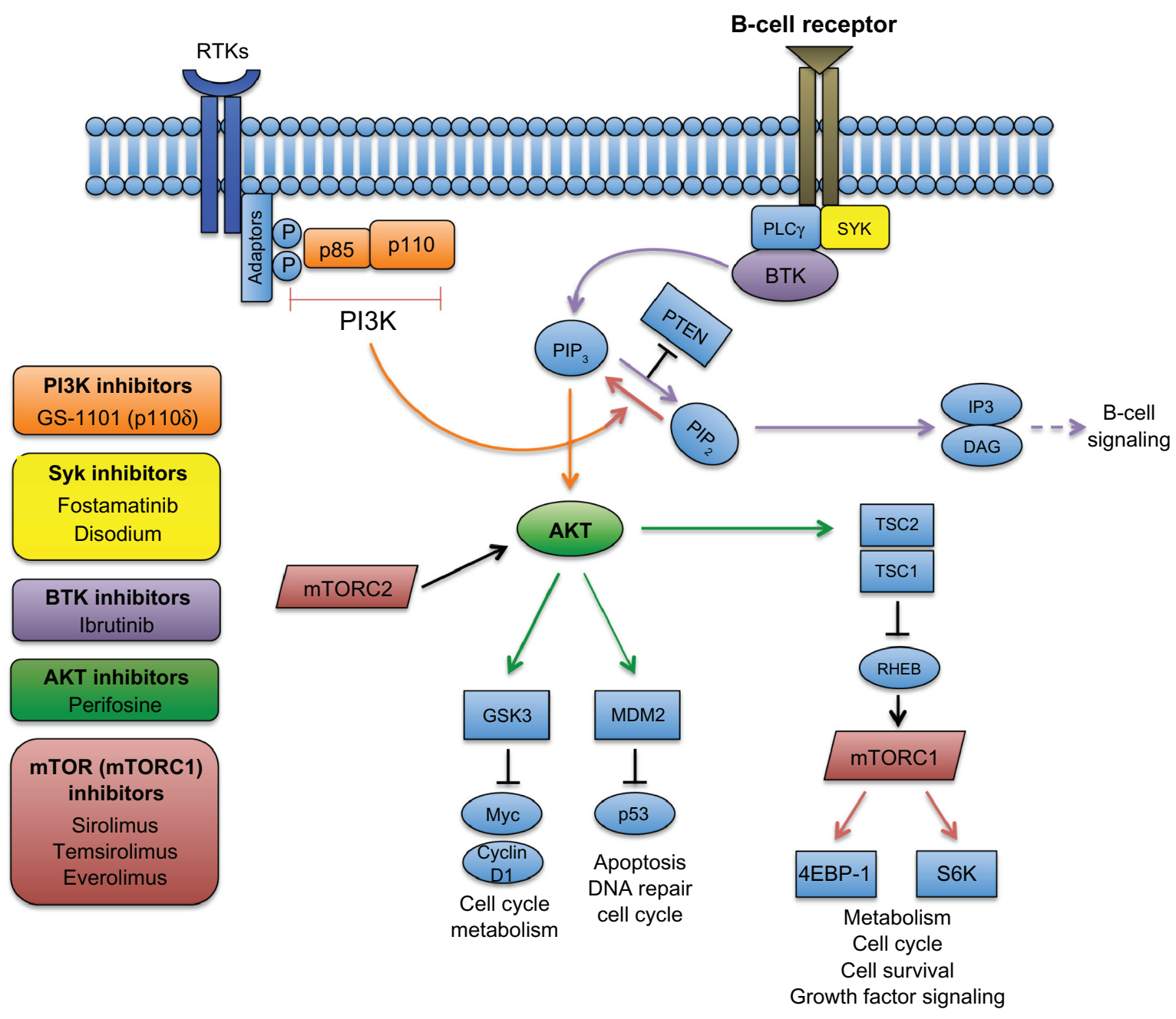

Figure 3 Targeting the PI3K and BTK pathways in NHL. The B-cell receptor-signaling pathway (purple) is initiated through phosphorylation of coreceptors that recruit spleen tyrosine kinase (SYK), which then phosphorylates downstream kinases, including PLC $\gamma$, leading to activation of Bruton tyrosine kinase (BTK). BTK then binds phosphatidylinositol $(3,4,5)$-triphosphate $\left(\mathrm{PIP}_{3}\right)$, which in turn hydrolyzes phosphatidylinositol 4,5-bisphosphate $\left(\mathrm{PIP}_{2}\right)$ into two second messengers - inositol triphosphate (IP3) and diacylglycerol (DAG) - that ultimately activate downstream proteins involved in B-cell signaling. In the PI3K pathway (orange), activation of receptor tyrosine kinases (RTKs) through adaptor proteins phosphorylate subunits of PI3K, leading to conversion of $\mathrm{PIP}_{2}$ to $\mathrm{PIP}_{3}$. Phosphorylation of the tumor suppressor PTEN terminates $\mathrm{PI} 3 \mathrm{~K}$. Accumulation of $\mathrm{PIP}_{3}$ leads to phosphorylation of AKT, which further activates downstream pathways, including $\mathrm{mTOR}$ (through the tuberous sclerosis complex I/2 $(\mathrm{TSCl} / 2)$ ) and other essential pathways. Therapeutic inhibition of select pathway components that have been investigated in clinical trials for NHL are shown.

Abbreviations: 4EBP-I, 4E-binding protein I; GSK3, glycogen synthase kinase 3; mTOR, mammalian target of rapamycin; mTORC, mTOR complex; MDM2, mouse double minute 2 homolog; P, phosphorylation site; PI3K, phosphoinositide 3-kinase; PLC, phospholipase C gamma; PTEN, phosphatase and tensin homolog; RHEB, Ras homolog enriched in brain; S6K, S6 kinase. 
This variable expression has led to the development of panPI3K isoform inhibitors. However, the ubiquitous expression and function of the multiple PI3K isoforms has also led to serious concerns about significant off-target toxicity. Therefore, isoform-specific inhibitors have also been explored. The $\mathrm{PI} 3 \mathrm{~K}-\delta$ isoform is selectively expressed in hematopoietic cells, thus creating a novel therapeutic target in B-cell malignancies. The oral PI3K- $\delta$ selective inhibitor CAL-101 (now renamed GS-1101) is perhaps the furthest developed, demonstrating selective apoptosis of CLL cells in preclinical models and clinical activity in indolent NHL subtypes. ${ }^{67}$ In phase I studies, GS-1101 was administered to patients with both refractory and relapsed indolent (FL, CLL) and aggressive (DLBCL, MCL) NHL (Table 2). ${ }^{68}$ Interim analyses in indolent NHL showed an ORR of $62 \%$, with mean duration of response not reached. For aggressive NHL subtypes, the ORR was also $62 \%$ in MCL, with median response duration of 3 months. There was no response in DLBCL patients. Reversible transaminitis was the only dose-limiting toxicity reported. Another phase I trial with relapsed/refractory CLL reported an ORR of $26 \%$, with $80 \%$ having a lymph-node response. ${ }^{69}$ Combination therapy with GS-1101 and rituximab and/or bendamustine in relapsed/refractory CLL has been investigated in a phase I trial. ${ }^{70} \mathrm{~A}$ favorable safety profile and lack of overlapping toxicities was observed. Virtually all patients had lymph-node reduction, with ORR for all regimens between $78 \%$ and $87 \%$. Additionally, GS-1101 was studied in combination with ofatumumab in refractory/relapsed CLL and achieved higher ORR and nodal response rates than single-agent therapy. ${ }^{71}$ Given the potent activity of GS-1101, other PI3K inhibitors are being investigated in NHL as single agents and as a combination strategy, with phase III studies planned. ${ }^{72}$

\section{AKT inhibitors}

AKT is a serine/threonine kinase that is directly activated in response to $\mathrm{PI} 3 \mathrm{~K}$, and is the PI3K effector that is most widely implicated in cancer. ${ }^{66}$ Activated AKT phosphorylates numerous substrates that promote growth, proliferation, and resistance to apoptosis (Figure 3), of which one immediate consequence is stimulation of mTOR (a therapeutic target discussed below). Of the selective AKT inhibitors, perifosine, an oral AKT inhibitor, has been the most characterized, with efficacy shown mainly in solid tumors and multiple myeloma. ${ }^{73}$ Results from a phase II trial combining perifosine and sorafenib (an oral multikinase inhibitor) in relapsed/ refractory NHL, Waldenström's macroglobulinemia, and HL achieved a partial response of $22 \%$, with a 4 -month median response duration (Table 2 ).${ }^{74}$ Of note, the majority of responders were patients with HL. Further clinical studies are needed to validate perifosine and other AKT inhibitors specifically in NHL with phase II trials under way.

\section{BTK inhibitors}

While AKT is the PI3K effector that is most widely implicated in cancer, there are several AKT-independent pathways activated by PI3K, which notably include BTK (Figure 3). BTK is a member of the Tec family of kinases, with a well-characterized role in B-cell receptor (BCR) signaling and B-cell activation. BTK is relatively restricted to hematopoietic cell types, with knockout studies in mice and man showing a predominant B-cell function. BTK mutations in humans give rise to X-linked agammaglobulinemia, an inherited disorder that leads to severely depressed levels of immunoglobulin production and absence of B cells. ${ }^{75}$ Because expression and knockout is largely restricted to B cells, BTK has been a uniquely attractive therapeutic target for malignancies relying on B-cell signaling. Ibrutinib (PCI32765), a selective and irreversible oral small-molecule BTK inhibitor, is the first of its class investigated in NHL. In vitro, ibrutinib is selectively cytotoxic to DLBCL cell lines driven by BCR signaling. ${ }^{76}$ The first phase I trial with ibrutinib in relapsed/refractory NHL was well tolerated, and showed an ORR of $60 \%$ across all histologies. Interestingly, CLL and MCL patients had a strong response rate (79\% and $75 \%$, respectively), while FL and DLBCL patients had a poor response (23\% and $17 \%$, respectively) (Table 2$).{ }^{77}$ Additionally, CLL patients noted rapid shrinkage of lymph nodes and marked lymphocytosis, which is thought to mark malignant cell egress from lymph nodes to peripheral blood. Given the specific high response rates and impressive lymph-node response, ibrutinib was further studied in CLL. Follow-up phase $\mathrm{Ib} / \mathrm{II}$ studies in relapsed/refractory CLL achieved an ORR of $67 \%$, with only minimal grade 3-4 hematologic toxicity. ${ }^{78}$ Ibrutinib was also evaluated in treatment-naïve CLL patients who were 65 years and older. ORR was $74 \%$, with $10 \%$ achieving CR and an estimated 15 -month PFS observed in an impressive $96 \%$ of patients. ${ }^{79}$ Compared to previously treated patients, the treatment-naïve patients showed faster response and higher ORR and CR rates. Given these high efficacy rates, ibrutinib was studied in combination strategies in phase I/II studies. When ibrutinib was combined with bendamustine and rituximab, ORR was $93 \%$, with $13 \%$ of patients achieving $\mathrm{CR} .{ }^{80}$ No dose reductions in ibrutinib were needed, with only a small fraction of patients requiring dose reductions of bendamustine. When ibrutinib was combined with ofatumumab, ORR was $100 \%{ }^{81}$

Ibrutinib appears to be well tolerated, with some unique adverse effects that are mostly platelet-mediated, including 

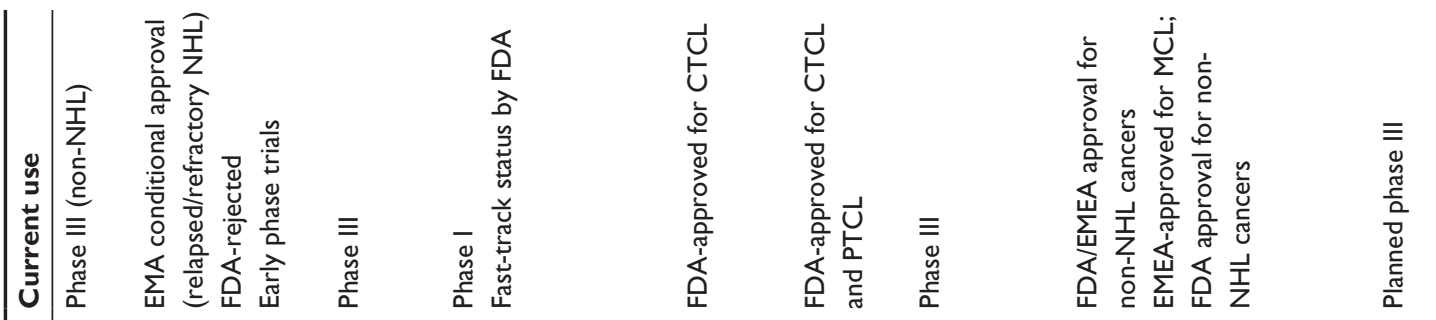

흐응으

$\infty \kappa$

요 $\infty$ 흐 으으

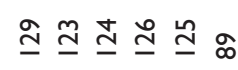

$\infty \quad \infty$

용

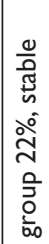

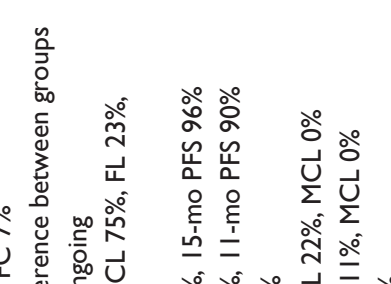

항 ㅇํㅇ

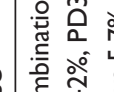

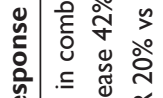

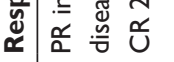

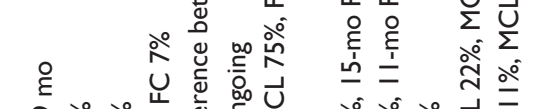

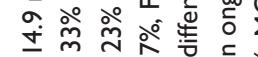

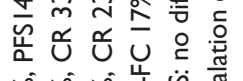

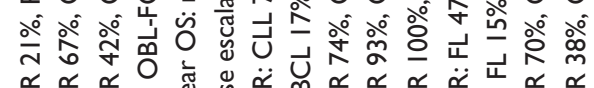

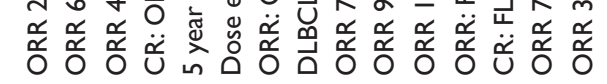

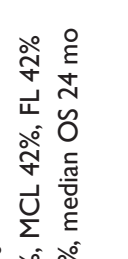

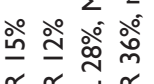

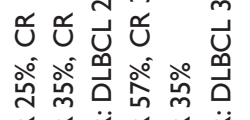

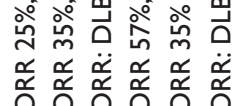

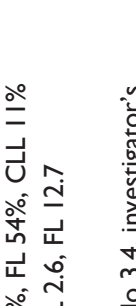

28

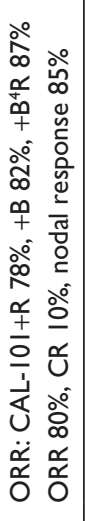

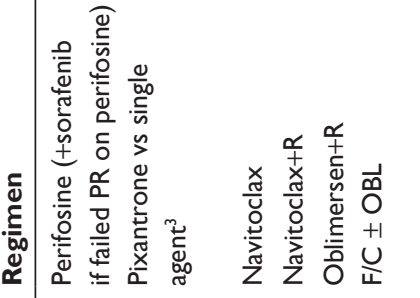

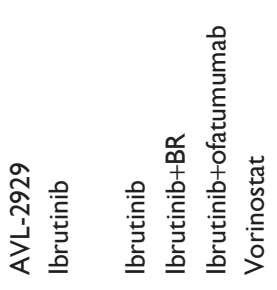

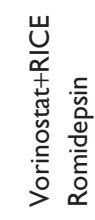

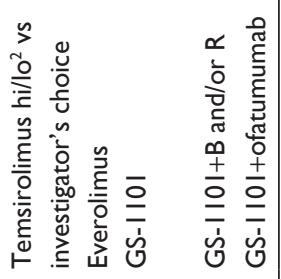

$\vec{U}$

$\stackrel{\overrightarrow{4}}{\vec{U}}\lrcorner \quad \vec{U}$

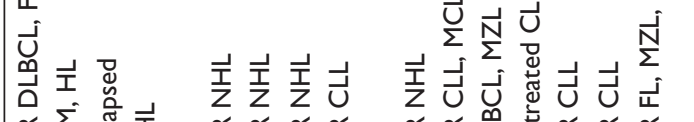

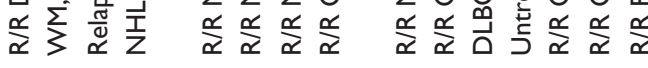

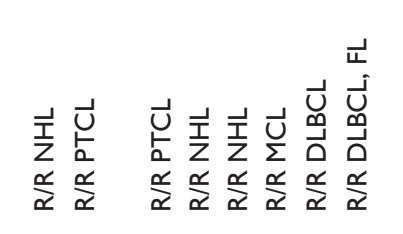

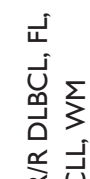

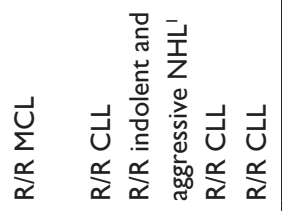

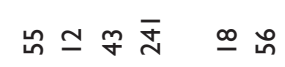

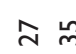

จิ คำำกำ

œ

$\overline{-}$ ส

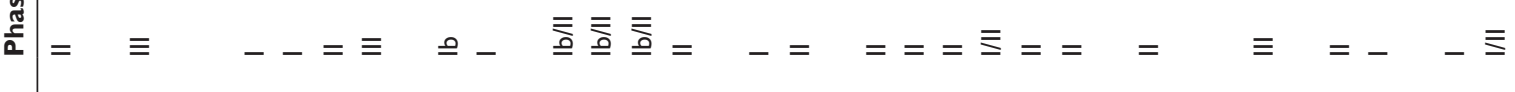




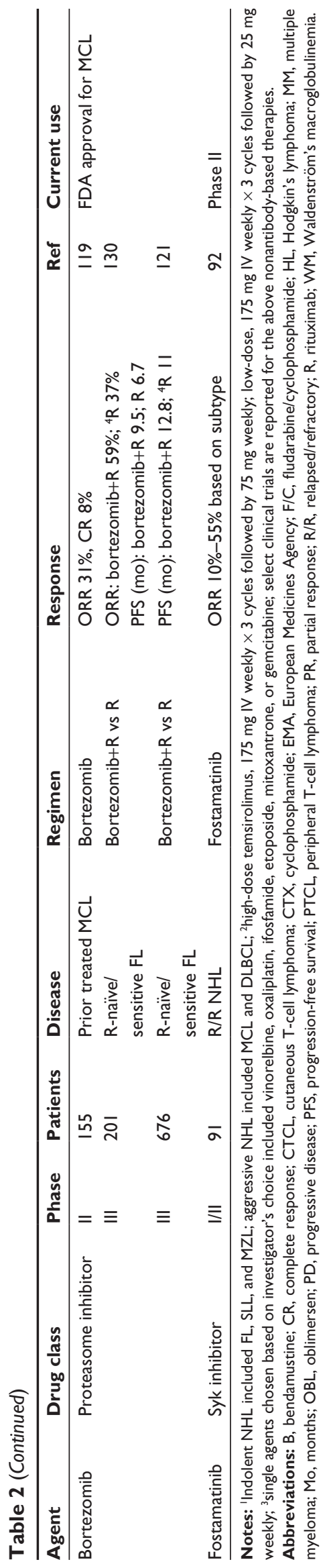

ecchymoses and rare occurrences of intracranial hemorrhage. These effects likely reflect the inhibition of platelet function through a direct effect on BTK. The clinical significance of this platelet inhibition is not completely understood; however, caution in combining ibrutinib with antiplatelet agents or other anticoagulants is warranted..$^{82}$ Nevertheless, ibrutinib has shown impressive clinical activity in early phase trials of CLL. Ibrutinib is one of few emerging therapies that have shown significant clinical activity against CLL and MCL, and has been given fast-track status by the FDA for accelerated approval for refractory disease. The success of ibrutinib has also spawned the generation of other BTK inhibitors, including the second-in-class inhibitor AVL-292, which is currently in a phase I study for previously treated NHL. ${ }^{83}$

\section{mTOR inhibitors}

One of the major effectors downstream of AKT is mTOR complex 1, which integrates many cellular inputs, including growth-factor signaling, metabolic state, cell survival, and proliferation (Figure 3). The mTOR complex has been therapeutically targeted by rapamycin derivatives, compounds first used in immunosuppression regimens and later in cancer. While rapamycin has been studied as an anticancer therapy, its low oral bioavailability and low solubility in aqueous solutions limited its development. ${ }^{84}$ This led to the development of temsirolimus, a naturally occurring water-soluble analog of rapamycin with improved bioavailability when administered intravenously. ${ }^{85}$ Temsirolimus, an intravenous prodrug that is metabolized to rapamycin, has been the most extensively evaluated in NHL. The efficacy of temsirolimus was first studied in MCL. MCL is differentiated by the expression of translocation $\mathrm{t}(11 ; 14)$ leading to deregulated overexpression of cyclin D1, which can be subsequently targeted by rapamycin analogs. ${ }^{86} \mathrm{~A}$ recent phase III study compared high- and low-dose temsirolimus to investigator's choice of prior approved therapy (Table 2) ${ }^{87}$ Higher dosing of temsirolimus outperformed both investigator's choice and lower-dose temsirolimus. However, ORR was $22 \%$, with a median PFS of 4.8 months. Nevertheless, temsirolimus is now being included in treatment guidelines as a therapeutic option for relapsed/refractory MCL. ${ }^{1}$ Temsirolimus has also been evaluated in other NHL subtypes. In a large phase II trial, temsirolimus was evaluated in non-MCL NHL. ${ }^{88}$ In DLBCL, ORR was $28.1 \%$, including $12.5 \%$ achieving $\mathrm{CR}$, but with a median PFS of only 2.6 months. In contrast, $53.8 \%$ of FL patients responded, including $25.6 \%$ achieving $\mathrm{CR}$, which led to a median PFS of 12.7 months. In CLL and other non-FL indolent lymphomas, only $11 \%$ of patients responded. 
As an alternative to temsirolimus, everolimus, an oral rapamycin analog, has also been evaluated in multiple NHL subtypes. The most recent phase II trial in DLBCL, MCL, and FL achieved an ORR of 30\%, with no major differences between the subtypes (Table 2).$^{89}$ In CLL, a separate phase II trial achieved a partial response of $18 \% .{ }^{90}$

With respect to toxicities, both temsirolimus and everolimus have similar profiles, with cytopenias being most common and pneumonitis being a concern in patients with a prior history of parenchymal lung disease. While both temsirolimus and everolimus demonstrate clinical response in several NHL subtypes (most notably in MCL), the relatively low response rates as a single agent may argue for these agents to be studied in combination with other therapies. Indeed, early stage trials are in progress for evaluating these rapamycin analogs with chemotherapies and targeted agents, including bendamustine, rituximab, sorafenib, and others. ${ }^{65}$

\section{Syk inhibitors}

Spleen tyrosine kinase (Syk) is involved in BCR signaling and initiates downstream signaling events after activation of the B-cell receptor (Figure 3). Syk expression and activity levels have been observed in various subtypes of NHL compared to normal B-cell counterparts. ${ }^{91}$ Fostamatinib disodium is an oral tyrosine kinase inhibitor whose active metabolite, R406, inhibits Syk at achievable plasma and intracellular concentrations. In a recent phase I/II study, relapsed/refractory NHL of various subtypes (DLBCL, FL, MCL, SLL/CLL) was treated with fostamatinib (Table 2). ${ }^{92}$ Of the various NHL subtypes, ORR ranged between $10 \%$ (for FL) up to 55\% for SLL/CLL. Neutropenia was the most frequently observed adverse event. Fostamatinib is currently in ongoing phase II trials for NHL. ${ }^{93,94}$

\section{Bcl-2 inhibitors}

The Bcl-2 family of proteins consists of proteins involved in pro- (Bax, Bak) and antiapoptotic (Bcl-2, Mcl-1) functions, and regulates the cellular balance between survival and cell death. Given the role of these proteins in cell survival, they have become attractive therapeutic targets in certain cancers. Overexpression of Bcl-2 is common in $\mathrm{NHL}$, and is associated with poor response to therapy and shortened survival. ${ }^{95}$ Additionally, overexpression of Bcl-2 has been demonstrated to render NHL subtypes resistant to rituximab-induced apoptosis. ${ }^{96,97}$ These observations and others have led to the development of Bcl-2 inhibitors for the treatment of NHL. Oblimersen is a Bcl-2 antisense molecule that targets and degrades Bcl-2 mRNA. In phase I trials in NHL, oblimersen had limited efficacy, ${ }^{98}$ but was generally well tolerated, with myelosuppresion observed as the most prevalent adverse event. When combined with rituximab, oblimersen achieved better response rates, with an ORR of $42 \%$ and a CR of $23 \%$ (Table 2 ) ${ }^{99}$ In the latest phase III trial, relapsed/refractory CLL patients were randomized to fludarabine/cyclophosphamide with or without oblimersen. The oblimersen group demonstrated superior ORR and 5-year median OS. ${ }^{100}$ Despite these results, the FDA is requiring a confirmatory trial prior to approval.

Navitoclax (ABT-263) is an orally bioavailable, highaffinity small molecule that inhibits the antiapoptotic activities of Bcl-2 and Bcl-XL. A phase I study in relapsed/ refractory NHL achieved an ORR of $21.7 \%$ with a median PFS of 14.9 months (Table 2). ${ }^{101}$ When combined with rituximab, navitoclax achieved an ORR of $67 \%$ with a CR of $33 \%$ in a small cohort of relapsed/refractory NHL patients. ${ }^{102}$ Significant toxicities included thrombocytopenia and lymphopenia, of which dose-limiting toxicities occurred, cautioning that unacceptable hematologic toxicities may occur with this drug.

\section{HDAC inhibitors}

Histone deacetylases (HDACs) are a class of enzymes that modify histones through acetylation, leading to changes in DNA expression. Reversible acetylation of proteins represents one of the most abundant posttranslational modifications in cells, and is achieved in great part by HDACs. Aberrant protein acetylation and abnormal expression of HDACs have been associated with human cancers. Increased expression of HDACs has been associated with poor prognosis, tumor invasion, and proliferative index in multiple cancer types. ${ }^{103}$ Thus, HDAC inhibitors (HDACis) have emerged as a novel anticancer drug class of great interest, and have shown preclinical and clinical efficacy. While HDACis are effective antiproliferative agents, the exact antineoplastic mechanisms are still unclear. They have been shown to mediate cell death through several pathways, including induction of cell-growth arrest and apoptosis, upregulation of tumor-suppressor genes, alteration of DNA repair and mitosis, induction of protein-folding response, and inhibition of angiogenesis. Currently, two HDACis, vorinostat and romidepsin, are clinically approved for the treatment of malignancies, specifically T-cell lymphomas. Clinical outcomes of vorinostat and romidepsin will be reviewed here. However, multiple HDACis are in clinical trials for other malignancies. 


\section{Vorinostat}

Vorinostat is an orally bioavailable HDACi with activity against class I and II deacetylases. While it is approved for CTCL, vorinostat has also been shown to have efficacy in other types of lymphoma. A phase II trial of patients with relapsed/refractory FL, MZL, and MCL treated with vorinostat achieved an ORR of $29 \%$ and CR of $14.5 \%$ (Table 2). ${ }^{104}$ Notably, response rates were significantly higher within FL patients, while no MCL patients responded to the study drug. Thus, vorinostat may have particular efficacy against FL. The median PFS was 15.6 months for FL, 5.9 months for MCL, and 18.8 months for MZL patients. Vorinostat was generally well tolerated, with the most common adverse events being cytopenias and fatigue. Given these results, vorinostat was investigated as a possible adjunct to standard salvage regimens in relapsed aggressive lymphomas as a novel therapeutic option in treating patients who could not receive dose escalation of traditional cytotoxic agents due to increased toxicity. In a phase I trial, vorinostat was added to rituximab + ICE (RICE) in relapsed and refractory NHL. ${ }^{105}$ Results were favorable, with the combination achieving a $70 \%$ ORR and a CR in $28 \%$ of patients. Results were particularly encouraging in DLBCL previously treated with rituximab, as response rates were $67 \%$ with vorinostat + RICE compared to $51 \%$ in rituximab-treated DLBCL patients treated with either RICE or rituximab + dexamethasone + cytarabine + cisplatin alone. ${ }^{106}$ Toxicities with the vorinostat combination regimen were comparable to RICE alone, with the exception of more frequent gastrointestinal toxicity and associated electrolyte abnormalities. While vorinostat has shown modest efficacy as a single agent, its therapeutic advantage lies in a combination strategy, given its mild toxicity profile and enhancement of chemotherapeutic efficacy.

\section{Romidepsin}

Romidepsin is a bicyclic HDACi that is approved for CTCL as well as PTCL, and has been mostly investigated in T-cell lymphomas. In a phase II trial of patients with relapsed/refractory PTCL, romidepsin achieved an ORR of $38 \%$ and a CR of $17 \%$, with a median duration of response of 8.9 months (Table 2). ${ }^{107}$ Common toxicities included thrombocytopenia, granulocytopenia, nausea, and fatigue. However, romidepsin was generally well tolerated, with no dose-limiting toxicities. A larger phase II trial in relapsed/ refractory PTCL with romidepsin as a single agent showed similar ORR and $\mathrm{CR}$ rates, with no significant dose-limiting toxicities.

\section{Novel chemotherapies \\ Pixantrone dimaleate}

While the anthracycline-containing regimen $\mathrm{R}-\mathrm{CHOP}$ is the cornerstone of first-line therapy for several NHL subtypes, anthracyclines have limited use in salvage regimens because of progressive dose-dependent cardiotoxicity that leads to progressive congestive heart failure. ${ }^{108}$ Pixantrone dimaleate (pixantrone) is an aza-anthracenedione anthracycline derivative that was synthesized approximately 20 years ago as an agent that provides similar antitumor properties to the anthracyclines, though with reduced cardiotoxicity. Because of structural modifications, pixantrone is less likely to generate reactive oxygen species or form alcohol metabolites, both of which are implicated in anthracycline-induced cardiotoxicity. ${ }^{109,110}$ Preclinical studies demonstrated markedly reduced cardiotoxicity with pixantrone when compared with doxorubicin or its analog mitoxantrone. ${ }^{111}$ In a phase I study in advanced or refractory NHL, pixantrone was tolerable at doses associated with antitumor activity, with grade 4 neutropenia as the sole dose-limiting toxicity. ${ }^{12}$ As a result, pixantrone was purposed as a therapy for NHL patients who had progressed or relapsed after anthracycline-containing treatment regimens. Subsequently, a phase III study was conducted to evaluate pixantrone as a single agent compared to the investigators' choice of single-agent chemotherapies in NHL patients who had relapsed after two or more prior chemotherapy regimens (one of which had contained an anthracycline) (Table 2). ${ }^{113}$ Importantly, a significant percentage of patients had not been previously treated with rituximab given its lack of availability at that time. Pixantrone-treated patients had a higher CR rate compared with the control group $(20 \%$ vs $5.7 \%$, respectively). Patients in the pixantrone group also experienced higher rates of neutropenia compared to controls ( $41.2 \%$ vs $19.4 \%$, respectively); however, this frequency was similar to prior anthracycline-based regimens. Of note, a higher frequency of cardiac adverse events was observed in the pixantrone group, which were mostly asymptomatic grade 1 and 2 declines in left ventricular ejection fraction with no evidence of dose-dependent cardiac toxicity. This observation may have been partially due to randomization effects, as five patients in the pixantrone group had histories of congestive heart failure or cardiomyopathy compared to none in the treatment group. Given these mixed results, the utility of pixantrone has been called into question. In 2010, the FDA denied pixantrone approval due to excessive neutropenia and cardiac toxicity as well as a lack of CR in the eight patients treated in the US part of the phase III trial. Despite the FDA's rejection, the European Medicines Agency 
granted drug approval in 2012 for NHL on the condition that pixantrone be further studied in patients who have received rituximab previously. While cardiotoxicity needs to be further investigated, pixantrone may represent an attractive singleagent therapy for refractory NHL.

\section{Bortezomib}

The proteasome is a protein complex that is chiefly involved in protein degradation, and represents another avenue for anticancer targeting. Proteasome inhibition has been shown to induce cell-cycle arrest and apoptosis, change cell-surface adhesion markers, and chemosensitize cells, leading to its development as a novel chemotherapy. ${ }^{114}$

Bortezomib, a proteasome inhibitor, has had particular success in the treatment of hematologic malignancies. It is FDA-approved for multiple myeloma and for MCL patients who have received at least one prior therapy. Bortezomib's activity in NHL may be in part due to inhibition of the nuclear factor- $\kappa \mathrm{B}$ signaling pathway, which is associated with Bcl-2 overexpression as well as activity on p53, which is commonly mutated in select NHL subtypes. ${ }^{114}$ Bortezomib in combination with rituximab or chemotherapies have been active and generally well tolerated in early phase trials in MCL, FL, and other subtypes of NHL. ${ }^{115-118}$ In MCL, patients receiving at least one prior therapy were treated with single-agent bortezomib, achieving an ORR of $31 \%$ and an $8 \%$ CR rate (Table 2). ${ }^{119}$ This multicenter phase II trial led to the FDA approval of bortezomib for the treatment of MCL patients with at least one prior therapy. Two phase III trials investigated bortezomib with rituximab vs rituximab alone for the treatment of relapsed, rituximab-sensitive/ naïve FL patients. Median PFS and ORR were higher in the bortezomib and rituximab group compared to rituximab alone. ${ }^{120,121}$ Regarding toxicity, common adverse events included neurologic complications and constipation, with peripheral neuropathies the most common event leading to discontinuation. Currently, bortezomib is a utilized secondline therapy for many MCL patients.

\section{Lenalidomide}

In contrast to other chemotherapies, lenalidomide is an immunomodulatory agent with several mechanisms of action, including direct antitumor effects, antiangiogenic properties, stimulation of immune effector cells, including cytotoxic $\mathrm{T}$ and natural killer cells, and modulation of the tumor microenvironment. ${ }^{122}$ Lenalidomide was first investigated in multiple myeloma and MDS, leading to FDA approval for both diseases. Lenalidomide has now been studied in NHL, with early phase trials demonstrating benefit in certain NHL subtypes. Phase II trials in aggressive relapsed/refractory NHL demonstrated an ORR of $35 \%$ for all subtypes, with CR rates between $12 \%$ and $13 \% .{ }^{123,124}$ ORR was higher in patients with MCL and FL compared to DLBCL. ${ }^{124}$ Significant toxicities were hematologic, with treatment-related cytopenias leading to dose reduction. Other toxicities included increased risk of thrombosis, hepatotoxicity, and teratogenicity. In addition to monotherapy, lenalidomide has also been studied in combination with rituximab in pretreated elderly DLBCL patients. ${ }^{125}$ ORR was $35 \%$, with $35 \%$ 1-year disease-free survival. In MCL, lenalidomide in combination with rituximab yielded an ORR of $57 \%$, with $36 \%$ of patients achieving CR. ${ }^{126}$ Given the observed activity in refractory patients and relatively well-tolerated toxicity profile, lenalidomide is currently being investigated in newly diagnosed patients who are not candidates for high-dose chemotherapy. ${ }^{127}$

\section{Conclusion}

Relapsed and refractory disease continues to represent the most significant challenge in treating NHL. While rituximab has markedly improved the prognosis of NHL, a significant number of patients are still refractory to rituximab-based therapies. Multiple therapeutic options exist for refractory disease, but the prognosis remains poor. However, in the last decade, several new classes of therapies have shown promise in improving the course of refractory disease. Antibody-based therapies have evolved considerably since the use of rituximab, with multiple next-generation anti-CD20 antibodies, antibody conjugates, and bispecific antibodies demonstrating significant efficacy. In addition, small-molecule inhibitors of PI3K and BTK, including GS-101 and ibrutinib, respectively, have demonstrated remarkable results in relapsed/refractory CLL and MCL, and are anticipated to gain FDA approval within the next year. The future of NHL therapy will continue to rely on the targeting of lymphoma-specific pathways through novel therapeutic approaches.

\section{Disclosure}

The author reports no conflicts of interest in this work.

\section{References}

1. Zelenetz AD, Abramson JS, Advani RH, et al. NCCN Clinical Practice Guidelines in Oncology: non-Hodgkin's lymphomas. J Natl Compr Canc Netw. 2010;8(3):288-334.

2. Jaffe ES, Harris NL, Stein H, Vardiman JW. World Health Organization Classification of Tumours: Pathology and Genetics of Tumours of Haematopoietic and Lymphoid Tissues. Vol 3, 3rd ed. Lyon: IARC; 2001. 
3. Coiffier B, Thieblemont C, Van Den Neste E, et al. Long-term outcome of patients in the LNH-98.5 trial, the first randomized study comparing rituximab-CHOP to standard CHOP chemotherapy in DLBCL patients: a study by the Groupe d'Etudes des Lymphomes de 1'Adulte. Blood. 2010;116(12):2040-2045.

4. Glennie MJ, French RR, Cragg MS, Taylor RP. Mechanisms of killing by anti-CD20 monoclonal antibodies. Mol Immunol. 2007;44(16): 3823-3837.

5. Davis TA, Grillo-López AJ, White CA, et al. Rituximab antiCD20 monoclonal antibody therapy in non-Hodgkin's lymphoma: safety and efficacy of re-treatment. J Clin Oncol. 2000;18(17):3135-3143.

6. Martín A, Conde E, Arnan M, et al. R-ESHAP as salvage therapy for patients with relapsed or refractory diffuse large B-cell lymphoma: the influence of prior exposure to rituximab on outcome. A GEL/TAMO study. Haematologica. 2008;93(12):1829-1836.

7. Hagberg H, Gisselbrecht C. Randomised phase III study of R-ICE versus R-DHAP in relapsed patients with CD20 diffuse large B-cell lymphoma (DLBCL) followed by high-dose therapy and a second randomisation to maintenance treatment with rituximab or not: an update of the CORAL study. Ann Oncol. 2006;17 Suppl 4:iv31-iv32.

8. Rezvani AR, Maloney DG. Rituximab resistance. Best Pract Res Clin Haematol. 2011;24(2):203-216.

9. Shimizu R, Kikuchi J, Wada T, Ozawa K, Kano Y, Furukawa Y. HDAC inhibitors augment cytotoxic activity of rituximab by upregulating CD20 expression on lymphoma cells. Leukemia. 2010;24(10): $1760-1768$

10. Moskowitz CH, Bertino JR, Glassman JR, et al. Ifosfamide, carboplatin, and etoposide: a highly effective cytoreduction and peripheralblood progenitor-cell mobilization regimen for transplant-eligible patients with non-Hodgkin's lymphoma. J Clin Oncol. 1999;17(12): 3776-3785.

11. Kewalramani T, Zelenetz AD, Nimer SD, et al. Rituximab and ICE as second-line therapy before autologous stem cell transplantation for relapsed or primary refractory diffuse large B-cell lymphoma. Blood. 2004;103(10):3684-3688.

12. Rai KR, Peterson BL, Appelbaum FR, et al. Fludarabine compared with chlorambucil as primary therapy for chronic lymphocytic leukemia. N Engl J Med. 2000;343(24):1750-1757.

13. Molica S. Progress in the treatment of chronic lymphocytic leukemia: results of the German CLL8 trial. Expert Rev Anticancer Ther. 2011; 11(9):1333-1340.

14. Catovsky D, Richards S, Matutes E, et al. Assessment of fludarabine plus cyclophosphamide for patients with chronic lymphocytic leukaemia (the LRF CLL4 Trial): a randomised controlled trial. Lancet. 2007;370(9583):230-239.

15. Dohner H, Stilgenbauer S, Benner A, et al. Genomic aberrations and survival in chronic lymphocytic leukemia. NEngl J Med. 2000;343(26): 1910-1916.

16. van Oers $M H$, Klasa R, Marcus RE, et al. Rituximab maintenance improves clinical outcome of relapsed/resistant follicular non-Hodgkin lymphoma in patients both with and without rituximab during induction: results of a prospective randomized phase 3 intergroup trial. Blood. 2006;108(10):3295-3301.

17. Hagemeister FB. Treatment of relapsed aggressive lymphomas: regimens with and without high-dose therapy and stem cell rescue. Cancer Chemother Pharmacol. 2002;49 Suppl 1:S13-S20.

18. Philip T, Guglielmi C, Hagenbeek A, et al. Autologous bone marrow transplantation as compared with salvage chemotherapy in relapses of chemotherapy-sensitive non-Hodgkin's lymphoma. $N$ Engl J Med. 1995;333(23):1540-1545.

19. Peniket AJ, Ruiz de Elvira MC, Taghipour G, et al. An EBMT registry matched study of allogeneic stem cell transplants for lymphoma: allogeneic transplantation is associated with a lower relapse rate but a higher procedure-related mortality rate than autologous transplantation. Bone Marrow Transplant. 2003;31(8):667-678.

20. Swann JB, Smyth MJ. Immune surveillance of tumors. J Clin Invest. 2007;117(5):1137-1146.
21. Appelbaum FR. Hematopoietic cell transplantation for non-Hodgkin's lymphoma: yesterday, today, and tomorrow. J Clin Oncol. 2008;26(18): 2927-2929.

22. Brown JR. The treatment of relapsed refractory chronic lymphocytic leukemia. Hematology Am Soc Hematol Educ Program. 2011;2011: $110-118$.

23. Mayes S, Brown N, Illidge TM. New antibody drug treatments for lymphoma. Expert Opin Biol Ther. 2011;11(5):623-640.

24. Cang S, Mukhi N, Wang K, Liu D. Novel CD20 monoclonal antibodies for lymphoma therapy. J Hematol Oncol. 2012;5:64.

25. Cheson BD. Ofatumumab, a novel anti-CD20 monoclonal antibody for the treatment of B-cell malignancies. J Clin Oncol. 2010;28(21): $3525-3530$.

26. Teeling JL, French RR, Cragg MS, et al. Characterization of new human CD20 monoclonal antibodies with potent cytolytic activity against non-Hodgkin lymphomas. Blood. 2004;104(6):1793-1800.

27. Gupta IV, Jewell RC. Ofatumumab, the first human anti-CD20 monoclonal antibody for the treatment of B cell hematologic malignancies. Ann N Y Acad Sci. 2012;1263:43-56.

28. Coiffier B, Lepretre S, Pedersen LM, et al. Safety and efficacy of ofatumumab, a fully human monoclonal anti-CD20 antibody, in patients with relapsed or refractory B-cell chronic lymphocytic leukemia: a phase 1-2 study. Blood. 2008;111(3):1094-1100.

29. Wierda WG, Kipps TJ, Mayer J, et al. Ofatumumab as single-agent CD20 immunotherapy in fludarabine-refractory chronic lymphocytic leukemia. J Clin Oncol. 2010;28(10):1749-1755.

30. Wierda WG, Kipps TJ, Durig J, et al. Chemoimmunotherapy with O-FC in previously untreated patients with chronic lymphocytic leukemia. Blood. 2011;117(24):6450-6458.

31. Czuczman MS, Fayad L, Delwail V, et al. Ofatumumab monotherapy in rituximab-refractory follicular lymphoma: results from a multicenter study. Blood. 2012;119(16):3698-3704

32. Czuczman MS, Hess G, Gadeberg OV, et al. Chemoimmunotherapy with ofatumumab in combination with CHOP in previously untreated follicular lymphoma. Br J Haematol. 2012;157(4): 438-445.

33. Morschhauser F, Marlton P, Vitolo U, et al. Results of a phase I/II study of ocrelizumab, a fully humanized anti-CD20 mAb, in patients with relapsed/refractory follicular lymphoma. Ann Oncol. 2010;21(9): 1870-1876.

34. Goldenberg DM, Morschhauser F, Wegener WA. Veltuzumab (humanized anti-CD20 monoclonal antibody): characterization, current clinical results, and future prospects. Leuk Lymphoma. 2010;51(5): 747-755.

35. Morschhauser F, Leonard JP, Fayad L, et al. Humanized anti-CD20 antibody, veltuzumab, in refractory/recurrent non-Hodgkin's lymphoma: phase I/II results. J Clin Oncol. 2009;27(20):3346-3353.

36. Tu X, LaVallee T, Lechleider R. CD22 as a target for cancer therapy. $J$ Exp Ther Oncol. 2011;9(3):241-248.

37. Leonard JP, Link BK. Immunotherapy of non-Hodgkin's lymphoma with hLL2 (epratuzumab, an anti-CD22 monoclonal antibody) and Hu1D10 (apolizumab). Semin Oncol. 2002;29(1 Suppl 2): 81-86.

38. Strauss SJ, Morschhauser F, Rech J, et al. Multicenter phase II trial of immunotherapy with the humanized anti-CD22 antibody, epratuzumab, in combination with rituximab, in refractory or recurrent non-Hodgkin's lymphoma. J Clin Oncol. 2006;24(24):3880-3886.

39. Gottlieb AB, Kang S, Linden KG, et al. Evaluation of safety and clinical activity of multiple doses of the anti-CD80 monoclonal antibody, galiximab, in patients with moderate to severe plaque psoriasis. Clin Immunol. 2004;111(1):28-37.

40. Vyth-Dreese FA, Boot H, Dellemijn TA, et al. Localization in situ of costimulatory molecules and cytokines in B-cell non-Hodgkin's lymphoma. Immunology. 1998;94(4):580-586.

41. Czuczman MS, Thall A, Witzig TE, et al. Phase I/II study of galiximab, an anti-CD80 antibody, for relapsed or refractory follicular lymphoma. J Clin Oncol. 2005;23(19):4390-4398. 
42. Czuczman MS, Leonard JP, Jung S, et al. Phase II trial of galiximab (anti-CD80 monoclonal antibody) plus rituximab (CALGB 50402): Follicular Lymphoma International Prognostic Index (FLIPI) score is predictive of upfront immunotherapy responsiveness. Ann Oncol. 2012;23(9):2356-2362.

43. Ishida $\mathrm{T}$, Utsunomiya $\mathrm{A}$, Iida $\mathrm{S}$, et al. Clinical significance of CCR4 expression in adult T-cell leukemia/lymphoma: its close association with skin involvement and unfavorable outcome. Clin Cancer Res. 2003;9(10 Pt 1):3625-3634.

44. Yamamoto K, Utsunomiya A, Tobinai K, et al. Phase I study of KW-0761, a defucosylated humanized anti-CCR4 antibody, in relapsed patients with adult T-cell leukemia-lymphoma and peripheral T-cell lymphoma. J Clin Oncol. 2010;28(9):1591-1598.

45. Ishida $\mathrm{T}$, Joh $\mathrm{T}$, Uike N, et al. Defucosylated anti-CCR4 monoclonal antibody (KW-0761) for relapsed adult T-cell leukemia-lymphoma: a multicenter phase II study. J Clin Oncol. 2012;30(8):837-842.

46. Advani A, Coiffier B, Czuczman MS, et al. Safety, pharmacokinetics, and preliminary clinical activity of inotuzumab ozogamicin, a novel immunoconjugate for the treatment of B-cell non-Hodgkin's lymphoma: results of a phase I study. J Clin Oncol. 2010;28(12):2085-2093.

47. Fayad L, Patel H, Verhoef G, et al. Safety and clinical activity of the anti-CD22 immunoconjugate inotuzumab ozogamicin (CMC-544) in combination with rituximab in follicular lymphoma or diffuse large B-cell lymphoma: preliminary report of a phase 1/2 study. ASH Annu Meet Abstr. 2008;112(11):266.

48. Clodi K, Younes A. Reed-Sternberg cells and the TNF family of receptors/ligands. Leuk Lymphoma. 1997;27(3-4):195-205.

49. Forero-Torres A, Leonard JP, Younes A, et al. A phase II study of SGN-30 (anti-CD30 mAb) in Hodgkin lymphoma or systemic anaplastic large cell lymphoma. Br J Haematol. 2009;146(2):171-179.

50. Blum KA, Jung SH, Johnson JL, et al. Serious pulmonary toxicity in patients with Hodgkin's lymphoma with SGN-30, gemcitabine, vinorelbine, and liposomal doxorubicin is associated with an FcgammaRIIIa-158 V/F polymorphism. Ann Oncol. 2010;21(11) 2246-2254.

51. Garnock-Jones KP. Brentuximab vedotin: a review of its use in patients with Hodgkin lymphoma and systemic anaplastic large cell lymphoma following previous treatment failure. Drugs. 2013;73(4):371-381.

52. Pro B, Advani R, Brice P, et al. Brentuximab vedotin (SGN-35) in patients with relapsed or refractory systemic anaplastic large-cell lymphoma: results of a phase II study. J Clin Oncol. 2012;30(18):2190-2196.

53. Viardot A, Goebeler M, Nopponey R, et al. Blinatumomab monotherapy shows efficacy in patients with relapsed diffuse large $\mathrm{B}$ cell lymphoma. ASH Annu Meet Abstr. 2011;188:1637.

54. Kaminski MS, Zelenetz AD, Press OW, et al. Pivotal study of iodine I 131 tositumomab for chemotherapy-refractory low-grade or transformed low-grade B-cell non-Hodgkin's lymphomas. J Clin Oncol. 2001;19(19):3918-3928.

55. Fisher RI, Kaminski MS, Wahl RL, et al. Tositumomab and iodine-131 tositumomab produces durable complete remissions in a subset of heavily pretreated patients with low-grade and transformed non-Hodgkin's lymphomas. J Clin Oncol. 2005;23(30):7565-7573.

56. Horning SJ, Younes A, Jain V, et al. Efficacy and safety of tositumomab and iodine-131 tositumomab (Bexxar) in B-cell lymphoma, progressive after rituximab. J Clin Oncol. 2005;23(4):712-719.

57. Witzig TE, Flinn IW, Gordon LI, et al. Treatment with ibritumomab tiuxetan radioimmunotherapy in patients with rituximab-refractory follicular non-Hodgkin's lymphoma. J Clin Oncol. 2002;20(15):3262-3269.

58. Gordon LI, Molina A, Witzig T, et al. Durable responses after ibritumomab tiuxetan radioimmunotherapy for CD20+ B-cell lymphoma: long-term follow-up of a phase 1/2 study. Blood. 2004;103(12): 4429-4431.

59. Press OW, Unger JM, Rimsza LM, et al. Phase III randomized intergroup trial of CHOP plus rituximab compared with CHOP chemotherapy plus (131)iodine-tositumomab for previously untreated follicular non-Hodgkin lymphoma: SWOG S0016. J Clin Oncol. 2013;31(3):314-320
60. Witzig TE, White CA, Gordon LI, et al. Safety of yttrium-90 ibritumomab tiuxetan radioimmunotherapy for relapsed low-grade, follicular, or transformed non-Hodgkin's lymphoma. J Clin Oncol. 2003;21(7): $1263-1270$.

61. Goldsmith SJ. Radioimmunotherapy of lymphoma: Bexxar and Zevalin. Semin Nucl Med. 2010;40(2):122-135.

62. Bennett JM, Kaminski MS, Leonard JP, et al. Assessment of treatmentrelated myelodysplastic syndromes and acute myeloid leukemia in patients with non-Hodgkin lymphoma treated with tositumomab and iodine I131 tositumomab. Blood. 2005;105(12):4576-4582.

63. Czuczman MS, Emmanouilides C, Darif M, et al. Treatment-related myelodysplastic syndrome and acute myelogenous leukemia in patients treated with ibritumomab tiuxetan radioimmunotherapy. J Clin Oncol. 2007;25(27):4285-4292.

64. Rodon J, Dienstmann R, Serra V, Tabernero J. Development of PI3K inhibitors: lessons learned from early clinical trials. Nat Rev Clin Oncol. 2013;10(3):143-153.

65. Schatz JH. Targeting the PI3K/AKT/mTOR pathway in non-Hodgkin's lymphoma: results, biology, and development strategies. Curr Oncol Rep. 2011;13(5):398-406.

66. Engelman JA. Targeting PI3K signalling in cancer: opportunities, challenges and limitations. Nat Rev Cancer. 2009;9(8):550-562.

67. Herman SE, Gordon AL, Wagner AJ, et al. Phosphatidylinositol 3-kinase-delta inhibitor CAL-101 shows promising preclinical activity in chronic lymphocytic leukemia by antagonizing intrinsic and extrinsic cellular survival signals. Blood. 2010;116(12):2078-2088.

68. Kahl B, Byrd JC, Flinn IW, et al. Clinical safety and activity in a phase 1 study of CAL-101, an isoform-selective inhibitor of phosphatidylinositol 3-kinase P110delta, in patients with relapsed or refractory non-Hodgkin lymphoma. ASH Ann Meet Abstr. 2010;166:1777.

69. Furman RR, Byrd JC, Brown JR, et al. CAL-101, an isoform-selective inhibitor of phosphatidylinositol 3-kinase P110delta, demonstrates clinical activity and pharmacodynamic effects in patients with relapsed or refractory chronic lymphocytic leukemia. ASH Ann Meet Abstr. 2010;116:55.

70. Coutre SE, Leonard JP, Furman RR, et al. Combinations of the selective phosphatidylinositol 3-kinase-delta (PI3 Kdelta) inhibitor GS-1101 (CAL-101) with rituximab and/or bendamustine are tolerable and highly active in patients with relapsed or refractory chronic lymphocytic leukemia (CLL): results from a phase I study. ASH Ann Meet Abstr. 2012;120:191

71. Furman RR, Barrientos JC, Sharman JP, et al. A phase I/II study of the selective phosphatidylinositol 3-kinase-delta (PI3 Kdelta) inhibitor, GS-1101 (CAL-101), with ofatumumab in patients with previously treated chronic lymphocytic leukemia (CLL). J Clin Oncol. 2012; 30 Suppl:6518.

72. Robak P, Robak T. A targeted therapy for protein and lipid kinases in chronic lymphocytic leukemia. Curr Med Chem. 2012;19(31): 5294-5318.

73. Richardson PG, Eng C, Kolesar J, Hideshima T, Anderson KC. Perifosine, an oral, anti-cancer agent and inhibitor of the Akt pathway: mechanistic actions, pharmacodynamics, pharmacokinetics, and clinical activity. Expert Opin Drug Metab Toxicol. 2012;8(5): 623-633.

74. Guidetti A, Viviani S, Marchiano A, et al. Dual targeted therapy with the AKT inhibitor perifosine and the multikinase inhibitor sorafenib in patients with relapsed/refractory lymphomas: final results of a phase II trial. ASH Аnпи Meet Abstr. 2012;120:3679.

75. Conley ME, Dobbs AK, Farmer DM, et al. Primary B cell immunodeficiencies: comparisons and contrasts. Annu Rev Immunol. 2009;27:199-227.

76. Davis RE, Ngo VN, Lenz G, et al. Chronic active B-cell-receptor signalling in diffuse large B-cell lymphoma. Nature. 2010;463(7277): 88-92.

77. Advani RH, Buggy JJ, Sharman JP, et al. Bruton tyrosine kinase inhibitor ibrutinib (PCI-32765) has significant activity in patients with relapsed/ refractory B-cell malignancies. J Clin Oncol. 2013;31(1):88-94. 
78. O'Brien S, Burger JA, Blum KA, et al. The Bruton's tyrosine kinase (BTK) inhibitor PCI-32765 induces durable responses in relapsed or refractory $(\mathrm{R} / \mathrm{R})$ chronic lymphocytic leukemia/small lymphocytic lymphoma (CLL/SLL): follow-up of a phase Ib/II study. ASH Annu Meet Abstr. 2011;118:983.

79. Byrd JC, Furman RR, Coutre SE, et al. The Bruton's tyrosine kinase (BTK) inhibitor PCI-32765 (P) in treatment-naive (TN) chronic lymphocytic leukemia (CLL) patients (pts): Interim results of a phase Ib/II study. J Clin Oncol. 2012;30 Suppl:6507.

80. O'Brien SM, Barrientos JC, Flinn IW, et al. Combination of the Bruton's tyrosine kinase (BTK) inhibitor PCI-32765 with bendamustine (B)/ rituximab $(\mathrm{R})(\mathrm{BR})$ in patients (Pts) with relapsed/refractory $(\mathrm{R} / \mathrm{R})$ chronic lymphocytic leukemia (CLL): interim results of a phase $\mathrm{Ib} / \mathrm{II}$ study J Clin Oncol. 2012;30 Suppl:6515.

81. Jaglowski SM, Jones JA, Flynn JM, et al. A phase Ib/II study evaluating activity and tolerability of BTK inhibitor PCI-32765 and ofatumumab in patients with chronic lymphocytic leukemia/small lymphocytic lymphoma (CLL/SLL) and related diseases. J Clin Oncol. 2012;30 Suppl: 6508.

82. Brown JR. Ibrutinib (PCI-32765), the first BTK (Bruton's tyrosine kinase) inhibitor in clinical trials. Curr Hematol Malig Rep. 2013;8(1): $1-6$.

83. Brown JR, Sharman JP, Harb WA, et al. Phase Ib trial of AVL-292, a covalent inhibitor of Bruton's tyrosine kinase (Btk), in chronic lymphocytic leukemia (CLL) and B-non-Hodgkin lymphoma (B-NHL). J Clin Oncol. 2012;30 Suppl:8032.

84. Kahan BD, Camardo JS. Rapamycin: clinical results and future opportunities. Transplantation. 2001;72(7):1181-1193.

85. Dudkin L, Dilling MB, Cheshire PJ, et al. Biochemical correlates of mTOR inhibition by the rapamycin ester CCI-779 and tumor growth inhibition. Clin Cancer Res. 2001;7(6):1758-1764.

86. Hipp S, Ringshausen I, Oelsner M, Bogner C, Peschel C, Decker T. Inhibition of the mammalian target of rapamycin and the induction of cell cycle arrest in mantle cell lymphoma cells. Haematologica. 2005;90(10):1433-1434.

87. Hess G, Herbrecht R, Romaguera J, et al. Phase III study to evaluate temsirolimus compared with investigator's choice therapy for the treatment of relapsed or refractory mantle cell lymphoma. J Clin Oncol. 2009;27(23):3822-3829.

88. Smith SM, van Besien K, Karrison T, et al. Temsirolimus has activity in non-mantle cell non-Hodgkin's lymphoma subtypes: the University of Chicago phase II consortium. J Clin Oncol. 2010;28(31):4740-4746.

89. Witzig TE, Reeder CB, LaPlant BR, et al. A phase II trial of the oral mTOR inhibitor everolimus in relapsed aggressive lymphoma. Leukemia. 2011;25(2):341-347.

90. Zent CS, LaPlant BR, Johnston PB, et al. The treatment of recurrent/ refractory chronic lymphocytic leukemia/small lymphocytic lymphoma (CLL) with everolimus results in clinical responses and mobilization of CLL cells into the circulation. Cancer. 2010;116(9):2201-2207.

91. Macias-Perez IM, Flinn IW. B-cell receptor pathobiology and targeting in NHL. Curr Oncol Rep. 2012;14(5):411-418.

92. Friedberg JW, Sharman J, Sweetenham J, et al. Inhibition of Syk with fostamatinib disodium has significant clinical activity in non-Hodgkin lymphoma and chronic lymphocytic leukemia. Blood. 2010;115(13): $2578-2585$.

93. AstraZeneca. Study to learn if $200 \mathrm{mg}$ test drug (fostamatinib) helps people with large B-cell lymphoma, a type of blood cancer. Bethesda, MD: US National Library of Medicine; 2011 [updated June 4, 2013]. Available from: http://clinicaltrials.gov/show/NCT01499303. NLM identifier: NCT01499303. Accessed June 17, 2013.

94. AstraZeneca. A randomised double blind phase II trial to evaluate the efficacy of two doses of fostamatinib in patients with relapsed or refractory diffuse large B-cell lymphoma (DLBCL). NLM identifier: NCT01499303.

95. Gascoyne RD, Adomat SA, Krajewski S, et al. Prognostic significance of $\mathrm{Bcl}-2$ protein expression and $\mathrm{Bcl}-2$ gene rearrangement in diffuse aggressive non-Hodgkin's lymphoma. Blood. 1997;90(1):244-251.
96. Stolz C, Hess G, Hahnel PS, et al. Targeting Bcl-2 family proteins modulates the sensitivity of B-cell lymphoma to rituximab-induced apoptosis. Blood. 2008;112(8):3312-3321.

97. Wobser M, Voigt H, Eggert AO, et al. Bcl-2 expression in rituximab refractory cutaneous B-cell lymphoma. Br J Cancer. 2007;96(10): $1540-1543$.

98. Waters JS, Webb A, Cunningham D, et al. Phase I clinical and pharmacokinetic study of bcl-2 antisense oligonucleotide therapy in patients with non-Hodgkin's lymphoma. J Clin Oncol. 2000;18(9): 1812-1823.

99. Pro B, Leber B, Smith M, et al. Phase II multicenter study of oblimersen sodium, a Bcl-2 antisense oligonucleotide, in combination with rituximab in patients with recurrent B-cell non-Hodgkin lymphoma. Br J Haematol. 2008;143(3):355-360.

100. O'Brien S, Moore JO, Boyd TE, et al. 5-Year survival in patients with relapsed or refractory chronic lymphocytic leukemia in a randomized, phase III trial of fludarabine plus cyclophosphamide with or without oblimersen. J Clin Oncol. 2009;27(31):5208-5212.

101. Wilson WH, O'Connor OA, Czuczman MS, et al. Navitoclax, a targeted high-affinity inhibitor of BCL-2, in lymphoid malignancies: a phase 1 dose-escalation study of safety, pharmacokinetics, pharmacodynamics, and antitumour activity. Lancet Oncol. 2010;11(12):1149-1159.

102. Kahl B, Roberts AW, Seymour JF, et al. Navitoclax (ABT-263) plus rituximab: interim results of a phase 1 study in patients with CD20-positive lymphoid malignancies. ASH Annu Meet Abstr. 2010;116:3943.

103. Tang J, Yan H, Zhuang S. Histone deacetylases as targets for treatment of multiple diseases. Clin Sci (Lond). 2013;124(11):651-662.

104. Kirschbaum M, Frankel P, Popplewell L, et al. Phase II study of vorinostat for treatment of relapsed or refractory indolent nonHodgkin's lymphoma and mantle cell lymphoma. J Clin Oncol. 2011;29(9):1198-1203.

105. Budde LE, Zhang MM, Shustov AR, et al. A phase I study of pulse high-dose vorinostat (V) plus rituximab (R), ifosphamide, carboplatin, and etoposide (ICE) in patients with relapsed lymphoma. Br $J$ Haematol. 2013;161(2):183-191.

106. Gisselbrecht C, Glass B, Mounier N, et al. Salvage regimens with autologous transplantation for relapsed large B-cell lymphoma in the rituximab era. J Clin Oncol. 2010;28(27):4184-4190.

107. Piekarz RL, Frye R, Prince HM, et al. Phase 2 trial of romidepsin in patients with peripheral T-cell lymphoma. Blood. 2011;117(22):5827-5834.

108. Swain SM, Whaley FS, Ewer MS. Congestive heart failure in patients treated with doxorubicin: a retrospective analysis of three trials. Cancer. 2003;97(11):2869-2879.

109. Salvatorelli E, Menna P, Paz OG, et al. The novel anthracenedione, pixantrone, lacks redox activity and inhibits doxorubicinol formation in human myocardium: insight to explain the cardiac safety of pixantrone in doxorubicin-treated patients. J Pharmacol Exp Ther. 2013;344(2): 467-478.

110. Minotti G, Menna P, Salvatorelli E, Cairo G, Gianni L. Anthracyclines: molecular advances and pharmacologic developments in antitumor activity and cardiotoxicity. Pharmacol Rev. 2004;56(2):185-229.

111. Cavalletti E, Crippa L, Mainardi P, et al. Pixantrone (BBR 2778) has reduced cardiotoxic potential in mice pretreated with doxorubicin: comparative studies against doxorubicin and mitoxantrone. Invest New Drugs. 2007;25(3):187-195.

112. Borchmann P, Schnell R, Knippertz R, et al. Phase I study of BBR 2778 , a new aza-anthracenedione, in advanced or refractory nonHodgkin's lymphoma. Ann Oncol. 2001;12(5):661-667.

113. Pettengell R, Coiffier B, Narayanan G, et al. Pixantrone dimaleate versus other chemotherapeutic agents as a single-agent salvage treatment in patients with relapsed or refractory aggressive nonHodgkin lymphoma: a phase 3, multicentre, open-label, randomised trial. Lancet Oncol. 2012;13(7):696-706.

114. Paoluzzi L, O'Connor OA. Mechanistic rationale and clinical evidence for the efficacy of proteasome inhibitors against indolent and mantle cell lymphomas. BioDrugs. 2006;20(1):13-23. 
115. Friedberg JW, Vose JM, Kelly JL, et al. The combination of bendamustine, bortezomib, and rituximab for patients with relapsed/ refractory indolent and mantle cell non-Hodgkin lymphoma. Blood. 2011;117(10):2807-2812.

116. Ribrag V, Gisselbrecht C, Haioun C, et al. Efficacy and toxicity of 2 schedules of frontline rituximab plus cyclophosphamide, doxorubicin, vincristine, and prednisone plus bortezomib in patients with B-cell lymphoma: a randomized phase 2 trial from the French Adult Lymphoma Study Group (GELA). Cancer. 2009;115(19):4540-4546.

117. de Vos S, Goy A, Dakhil SR, et al. Multicenter randomized phase II study of weekly or twice-weekly bortezomib plus rituximab in patients with relapsed or refractory follicular or marginal-zone B-cell lymphoma. J Clin Oncol. 2009;27(30):5023-5030.

118. Agathocleous A, Rohatiner A, Rule S, et al. Weekly versus twice weekly bortezomib given in conjunction with rituximab, in patients with recurrent follicular lymphoma, mantle cell lymphoma and Waldenström macroglobulinaemia. Br J Haematol. 2010;151(4): 346-353.

119. Fisher RI, Bernstein SH, Kahl BS, et al. Multicenter phase II study of bortezomib in patients with relapsed or refractory mantle cell lymphoma. J Clin Oncol. 2006;24(30):4867-4874.

120. Zinzani PL, Pellegrini C, Merla E, et al. Bortezomib as salvage treatment for heavily pretreated relapsed lymphoma patients: a multicenter retrospective study. Hematol Oncol. Epub October 29, 2012.

121. Coiffier B, Osmanov EA, Hong X, et al. Bortezomib plus rituximab versus rituximab alone in patients with relapsed, rituximab-naive or rituximab-sensitive, follicular lymphoma: a randomised phase 3 trial. Lancet Oncol. 2011;12(8):773-784.

122. Chiappella A, Vitolo U. Lenalidomide in diffuse large B-cell lymphomas. Adv Hematol. 2012;2012:498342.

123. Wiernik PH, Lossos IS, Tuscano JM, et al. Lenalidomide monotherapy in relapsed or refractory aggressive non-Hodgkin's lymphoma. J Clin Oncol. 2008;26(30):4952-4957.
124. Witzig TE, Vose JM, Zinzani PL, et al. An international phase II trial of single-agent lenalidomide for relapsed or refractory aggressive B-cell non-Hodgkin's lymphoma. Ann Oncol. 2011;22(7):1622-1627.

125. Zinzani PL, Pellegrini C, Gandolfi L, et al. Combination of lenalidomide and rituximab in elderly patients with relapsed or refractory diffuse large B-cell lymphoma: a phase 2 trial. Clin Lymphoma Myeloma Leuk. 2011;11(6):462-466.

126. Wang M, Fayad L, Wagner-Bartak N, et al. Lenalidomide in combination with rituximab for patients with relapsed or refractory mantle-cell lymphoma: a phase 1/2 clinical trial. Lancet Oncol. 2012;13(7): 716-723.

127. Chiappella A, Franceschetti S, Castellino A, Carella AM, et al. Rituximab-CHOP21 plus lenalidomide (LR-CHOP21) is effective and feasible in elderly untreated diffuse large B-cell lymphoma (DLBCL): results of phase II REAL07 study of the Fondazione Italiana Linfomi (FIL). Presented at: 54th ASH Annual Meeting and Exposition; December 8-11, 2012; Atlanta, GA, USA.

128. Micallef IN, Maurer MJ, Wiseman GA, et al. Epratuzumab with rituximab, cyclophosphamide, doxorubicin, vincristine, and prednisone chemotherapy in patients with previously untreated diffuse large B-cell lymphoma. Blood. 2011;118(15):4053-4061.

129. Coiffier B, Pro B, Prince HM, et al. Results from a pivotal, open-label, phase II study of romidepsin in relapsed or refractory peripheral T-cell lymphoma after prior systemic therapy. J Clin Oncol. 2012;30(6) 631-636.

130. Zinzani PL, Khuageva NK, Wang H, et al. Bortezomib plus rituximab versus rituximab in patients with high-risk, relapsed, rituximab-naive or rituximab-sensitive follicular lymphoma: subgroup analysis of a randomized phase 3 trial. J Hematol Oncol. 2012;5:67.

131. National Comprehensive Cancer Network (NCCN). NCCN Clinical Practice Guidelines in Oncology. Non-Hodgkin's Lymphomas Version 1.2013. Available from: http://www.nccn.org/professionals/ physician_gls/f_guidelines.asp.
Cancer Management and Research

\section{Publish your work in this journal}

Cancer Management and Research is an international, peer-reviewed open access journal focusing on cancer research and the optimal use of preventative and integrated treatment interventions to achieve improved outcomes, enhanced survival and quality of life for the cancer patient The journal welcomes original research, clinical \& epidemiological

\section{Dovepress}

studies, reviews \& evaluations, guidelines, expert opinion \& commentary, case reports \& extended reports. The manuscript management system is completely online and includes a very quick and fair peerreview system, which is all easy to use. Visit http://www.dovepress.com/ testimonials.php to read real quotes from published authors. 